\title{
Output-Only Modal Parameter Recursive Estimation of Time-Varying Structures via a Kernel Ridge Regression FS-TARMA Approach
}

\author{
Zhi-Sai Ma, Li Liu, Si-Da Zhou, and Lei Yu \\ School of Aerospace Engineering, Beijing Institute of Technology, Zhongguancun South Street 5, Beijing 100081, China \\ Correspondence should be addressed to Si-Da Zhou; zhousida@bit.edu.cn
}

Received 21 July 2016; Revised 2 November 2016; Accepted 29 November 2016; Published 11 January 2017

Academic Editor: Lu Chen

Copyright (C) 2017 Zhi-Sai Ma et al. This is an open access article distributed under the Creative Commons Attribution License, which permits unrestricted use, distribution, and reproduction in any medium, provided the original work is properly cited.

Modal parameter estimation plays an important role in vibration-based damage detection and is worth more attention and investigation, as changes in modal parameters are usually being used as damage indicators. This paper focuses on the problem of output-only modal parameter recursive estimation of time-varying structures based upon parameterized representations of the time-dependent autoregressive moving average (TARMA). A kernel ridge regression functional series TARMA (FS-TARMA) recursive identification scheme is proposed and subsequently employed for the modal parameter estimation of a numerical threedegree-of-freedom time-varying structural system and a laboratory time-varying structure consisting of a simply supported beam and a moving mass sliding on it. The proposed method is comparatively assessed against an existing recursive pseudolinear regression FS-TARMA approach via Monte Carlo experiments and shown to be capable of accurately tracking the time-varying dynamics in a recursive manner.

\section{Introduction}

The need for damage identification or fault diagnosis of a structure is pervasive throughout the aerospace, shipbuilding, manufacturing, infrastructure, and transportation engineering communities. In recent years, many efforts have been undertaken to detect, locate, and characterize damage in structural systems by examining changes in measured vibration responses, which may be collectively referred to as vibration-based damage identification or fault diagnosis [1-5]. Compared with visual or localized methods, vibrationbased methods offer a number of potential advantages, as they require no visual inspection, are capable of working at a "system level" especially when the portion of the structure being inspected is not accessible, and can monitor a structure under its operating conditions [1-5]. The basic idea behind this technology is that changes in the physical properties of a structure will cause detectable changes in its modal parameters (natural frequencies, damping ratios, and mode shapes), as the modal parameters are functions of the physical properties of the structure (mass, damping, and stiffness) [1].
Because changes in modal parameters are usually being used as damage indicators, modal parameter estimation plays an important role in vibration-based damage detection of structures and is worth more attention and investigation.

Most structures in the real world are time-varying under a specific time scale and their intrinsic time-varying behavior is increasingly inevitable in industry. Typical examples include vibration absorbers with variable stiffness, bridges with crossing vehicles, launch vehicles with varying fuel mass, airplanes with varying additional aerodynamic effects in flight, deployable space structures, and rotating machinery. In contrast to time-invariant systems producing stationary responses with time-invariant statistical characteristics [6], timevarying systems exhibit time-varying/nonstationary characteristics, requiring time-dependent dynamic models and corresponding identification methods. Therefore, time-varying system identification becomes increasingly more important [7-12].

This paper focuses on the problem of output-only modal parameter recursive estimation of time-varying structures due to the following two reasons. Firstly, in many cases 
controlled testing may not be feasible or the excitation may be unobservable under realistic operating conditions. Hence, there exists a need to estimate modal parameters of timevarying structures by exclusively using the available measured vibration responses (referred to as output-only modal parameter estimation). Secondly, in many practical cases in which the data observations are arriving in a continuous stream, the structure has to be identified before all of the response measurements are known. Hence, there also exists a need to estimate modal parameters of time-varying structures in a recursive instead of batch manner.

Many output-only identification methods have been developed based on parameterized representations of the time-dependent autoregressive moving average (TARMA) [10-14]. TARMA model-based identification methods are divided into three main classes, depending on the type of "structure" imposed upon the evolution of the time-varying model parameters [11, 12]: unstructured parameter evolution (UPE) methods, stochastic parameter evolution (SPE) methods, and deterministic parameter evolution (DPE) methods. Most of the output-only recursive identification methods are currently of the UPE type [9-13], which may be not suitable for fast varying structures as they impose no "structure" upon their model parameters [10-12]. These methods include the recursive maximum likelihood TARMA method [9-11] and the recursive pseudolinear regression TARMA (RPLRTARMA) method $[9,10,13]$. In contrast with the UPE/SPE methods, the DPE methods are based on explicit models of the parameter variation. These models are achieved by approximating the parameter trajectory by a linear combination of known basis functions, belonging to specific functional subspaces. Such representations are generally referred to as functional series TARMA (FS-TARMA) representations/models [11-14]. Although different recursive DPE methods have been developed in recent years, their tracking capability of time-varying dynamics may be limited and more efforts are still needed [10-20]. From this point of view the FSTARMA model-based recursive methods are promising and are therefore investigated in this work.

The remainder of the paper is organized as follows: Section 2 introduces the FS-TARMA model-based modal parameter estimation. The existing recursive pseudolinear regression FS-TARMA (RPLR-FS-TARMA) approach is reviewed in Section 3. Section 4 proposes a kernel ridge regression FSTARMA (KRR-FS-TARMA) approach consisting of parameter matrix estimation and the corresponding model structure selection. The proposed method is, along with the existing RPLR-FS-TARMA method, numerically and experimentally validated in Sections 5 and 6, respectively. Section 7 summarizes the study.

\section{FS-TARMA Model-Based Modal Parameter Estimation}

2.1. TARMA Model. The TARMA representation resembles its stationary counterparts, with the significant difference being that it allows its parameters to depend upon time [10-12]. A TARMA $\left(n_{a}, n_{c}\right)$ model, with $n_{a}, n_{c}$ designating, respectively, its autoregressive (AR) and moving average (MA) orders, is of the form [13]

$$
\begin{aligned}
\mathbf{x}[t]+\sum_{i=1}^{n_{a}} \mathbf{A}_{i}[t] \mathbf{x}[t-i]=\mathbf{e}[t]+\sum_{i=1}^{n_{c}} \mathbf{C}_{i}[t] & \mathbf{e}[t-i] \\
\mathbf{e}[t] & \sim \mathcal{N}(\mathbf{0}, \mathbf{\Sigma}[t])
\end{aligned}
$$

with $t$ designating normalized (by the sampling period) discrete time, $\mathbf{x}[t] \in \mathbb{R}^{k \times 1}$ the discrete-time nonstationary vibration signal to be modeled, and $\mathbf{e}[t] \in \mathbb{R}^{k \times 1}$ an unobservable uncorrelated innovation (residual) sequence with zero mean and time-varying nonsingular covariance matrix $\Sigma[t] \epsilon$ $\mathbb{R}^{k \times k} . \mathbf{A}_{i}[t]$ and $\mathbf{C}_{i}[t]$ are, respectively, the model's $A R$ and MA parameter matrices. $\mathcal{N}(\cdot, \cdot)$ stands for normally independently distributed random variables with the indicated mean and covariance.

Once the time-dependent $\mathrm{AR}$ parameter matrix $\mathbf{A}_{i}[t]$ has been obtained, the system's "frozen-time" poles can be derived by solving the following general eigenvalue problem [14]

$$
\left(\mathbf{D}[t]-p_{r}[t] \mathbf{I}_{k n_{a}}\right) \mathbf{V}_{r}[t]=0, \quad r=1,2, \ldots, k n_{a},
$$

where $\mathbf{I}_{k n_{a}}$ designates the identity matrix of order $k n_{a}$ and $p_{r}[t]$ and $\mathbf{V}_{r}[t]=\left[p_{r}[t]^{-n_{a}} \mathbf{L}_{r}^{T}, \ldots, p_{r}[t]^{-1} \mathbf{L}_{r}^{T}\right]^{T}$ are the $r$ th pole and eigenvector of $\mathbf{D}[t]$ at instant of time $t$ with the $r$ th mode shape $\mathbf{L}_{r}$, respectively. The matrix $\mathbf{D}[t]$ is constructed from the AR parameter matrices as

$$
\mathbf{D}[t]=\left[\begin{array}{cccc}
\mathbf{0} & \mathbf{I}_{k} & \cdots & \mathbf{0} \\
\vdots & \vdots & \ddots & \vdots \\
\mathbf{0} & \mathbf{0} & \cdots & \mathbf{I}_{k} \\
-\mathbf{A}_{n_{a}}[t] & -\mathbf{A}_{n_{a}-1}[t] & \cdots & -\mathbf{A}_{1}[t]
\end{array}\right] .
$$

The system's "frozen-time" modes with natural frequencies and damping ratios are computed by [6]

$$
\begin{aligned}
& f_{r}[t]= \frac{\left|\ln p_{r}[t]\right|}{2 \pi \Delta t}, \\
& \zeta_{r}[t]=\frac{-\operatorname{Re}\left(\ln p_{r}[t]\right)}{\left|\ln p_{r}[t]\right|}, \\
& r=1,2, \ldots, k n_{a}
\end{aligned}
$$

in which $|\cdot|$ denotes the absolute value and $\Delta t$ the sampling period.

2.2. FS-TARMA Model. Any given function can be approximated by a set of selected basis functions with arbitrary accuracy, as long as a sufficient number of basis functions are used [21]. Hence, a time-varying parameter $v[t]$ can be expressed as

$$
v[t]=\sum_{j=1}^{p_{v}} v^{j} \cdot G_{b_{v}(j)}[t],
$$


where $G_{b_{v}(j)}[t]$ denotes a set of basis functions selected from a suitable functional subspace (such as trigonometric, Legendre, Chebyshev, wavelets, B-splines, and moving Kriging shape functions), $v^{j}$ coefficient of projection, $p_{v}$ the subspace dimensionality, and the index $b_{v}(i)\left(i=1, \ldots, p_{v}\right)$ the specific basis functions of the selected functional subspace that are included in the basis.

By using (5), the time-dependent AR and MA parameter matrices $\mathbf{A}_{i}[t]$ and $\mathbf{C}_{i}[t]$ can be, respectively, expressed as [1114]

$$
\begin{array}{r}
\mathbf{A}_{i}[t] \approx\left[\begin{array}{ccc}
\sum_{j=1}^{p_{a}} a_{1,1}^{i, j} \cdot G_{b_{a}(j)}[t] & \cdots & \sum_{j=1}^{p_{a}} a_{1, k}^{i, j} \cdot G_{b_{a}(j)}[t] \\
\vdots & \ddots & \vdots \\
\sum_{j=1}^{p_{a}} a_{k, 1}^{i, j} \cdot G_{b_{a}(j)}[t] & \cdots & \sum_{j=1}^{p_{a}} a_{k, k}^{i, j} \cdot G_{b_{a}(j)}[t]
\end{array}\right], \\
\mathbf{C}_{i}[t] \approx\left[\begin{array}{ccc}
\sum_{j=1}^{p_{c}} c_{1,1}^{i, j} \cdot G_{b_{c}(j)}[t] & \cdots & \sum_{j=1}^{p_{c}} c_{1, k}^{i, j} \cdot G_{b_{c}(j)}[t] \\
\vdots & \ddots & \vdots \\
\sum_{j=1}^{p_{c}} c_{k, 1}^{i, j} \cdot G_{b_{c}(j)}[t] & \cdots & \sum_{j=1}^{p_{c}} c_{k, k}^{i, j} \cdot G_{b_{c}(j)}[t]
\end{array}\right] .
\end{array}
$$

Substituting (6) into (1), the TARMA model can be rewritten into the following FS-TARMA model:

$$
\begin{aligned}
& \mathbf{x}[t]=-\sum_{i=1}^{n_{a}} \mathbf{A}_{i}[t] \mathbf{x}[t-i]+\sum_{i=1}^{n_{c}} \mathbf{C}_{i}[t] \mathbf{e}[t-i]+\mathbf{e}[t] \Longrightarrow \\
& \mathbf{x}[t]=\boldsymbol{\theta}^{T} \boldsymbol{\phi}[t]+\mathbf{e}[t]
\end{aligned}
$$

with

$$
\begin{aligned}
\boldsymbol{\theta} & =\left[\begin{array}{ccccccc}
a_{1,1}^{1,1} & \cdots & a_{1,1}^{1, p_{a}} & \cdots & c_{1, k}^{n_{c}, 1} & \cdots & c_{1, k}^{n_{c}, p_{c}} \\
& \vdots & & \ddots & & \vdots & \\
a_{k, 1}^{1,1} & \cdots & a_{k, 1}^{1, p_{a}} & \cdots & c_{k, k}^{n_{c}, 1} & \cdots & c_{k, k}^{n_{c}, p_{c}}
\end{array}\right]^{T} \\
& \in \mathbb{R}^{k\left(n_{a} p_{a}+n_{c} p_{c}\right) \times k}
\end{aligned}
$$

being the time-invariant parameter matrix, and

$$
\phi[t]=\left[\begin{array}{c}
-\mathbf{x}[t-1] \otimes \mathbf{g}_{b_{a}}[t] \\
\vdots \\
-\mathbf{x}\left[t-n_{a}\right] \otimes \mathbf{g}_{b_{a}}[t] \\
\mathbf{e}[t-1] \otimes \mathbf{g}_{b_{c}}[t] \\
\vdots \\
\mathbf{e}\left[t-n_{c}\right] \otimes \mathbf{g}_{b_{c}}[t]
\end{array}\right] \in \mathbb{R}^{k\left(n_{a} p_{a}+n_{c} p_{c}\right) \times 1}
$$

the corresponding regression vector, where $\otimes$ is the Kronecker product [22] and $\mathbf{g}_{b_{a}}[t]$ and $\mathbf{g}_{b_{c}}[t]$ are, respectively, given by

$$
\begin{aligned}
\mathbf{g}_{b_{a}}[t] & =\left[G_{b_{a}(1)}[t], G_{b_{a}(2)}[t], \ldots, G_{b_{a}\left(p_{a}\right)}[t]\right]^{T} \\
& \in \mathbb{R}^{p_{a} \times 1}, \\
\mathbf{g}_{b_{c}}[t] & =\left[G_{b_{c}(1)}[t], G_{b_{c}(2)}[t], \ldots, G_{b_{c}\left(p_{c}\right)}[t]\right]^{T} \\
& \in \mathbb{R}^{p_{c} \times 1} .
\end{aligned}
$$

Unlike the TARMA model of (1), the parameter matrix of the FS-TARMA model of (7) is time-invariant; that is, $\boldsymbol{\theta}$ is a time-invariant matrix. In other words, a time-varying TARMA model can be transformed into a time-invariant FS-TARMA model by expanding the time-dependent model parameters onto the selected functional subspaces. The parameter matrix $\boldsymbol{\theta}$ may be thus estimated by means of timeinvariant system identification techniques, while the timedependent $\mathrm{AR}$ and MA parameter matrices $\mathbf{A}_{i}[t]$ and $\mathbf{C}_{i}[t]$ may be subsequently estimated by using (6), after substituting $\boldsymbol{\theta}$ by the obtained estimate $\widehat{\boldsymbol{\theta}}$.

\section{Recursive Pseudolinear Regression FS-TARMA Approach}

So far, we have presented that modal parameters can be extracted from the FS-TARMA model parameter matrix estimate $\widehat{\boldsymbol{\theta}}$. The problem of FS-TARMA model parameter matrix estimation based on the existing recursive pseudolinear regression scheme is reviewed in this section.

3.1. RPLR-FS-TARMA Method. The FS-TARMA model is time-invariant, as shown in (7), and the well-known recursive pseudolinear regression scheme $[9,10]$ can be utilized to accomplish the estimation of $\boldsymbol{\theta}$. Given the regularization parameter $\gamma \geq 0$, the regularized least squares cost function is defined as

$$
\min _{\boldsymbol{\theta}}\left[\frac{1}{2} \sum_{\tau=1}^{t}\left\|\mathbf{x}[\tau]-\boldsymbol{\theta}^{T} \boldsymbol{\phi}[\tau]\right\|^{2}+\frac{1}{2} \gamma\|\boldsymbol{\theta}\|^{2}\right]
$$

where $\|\cdot\|$ denotes the Euclidean norm. The RPLR-FSTARMA method can be obtained by minimizing the cost function of (12) and is summarized in the following [11]: 


$$
\begin{aligned}
\text { Prediction error: } & \widehat{\mathbf{e}}[t \mid t-1]=\mathbf{x}[t]-\widehat{\mathbf{x}}[t \mid t-1]=\mathbf{x}[t]-\widehat{\boldsymbol{\theta}}[t-1]^{T} \boldsymbol{\phi}[t], \\
\text { Gain: } & \mathbf{K}[t]=\mathbf{P}[t-1] \boldsymbol{\phi}[t]\left(1+\phi[t]^{T} \mathbf{P}[t-1] \boldsymbol{\phi}[t]\right)^{-1}, \\
\text { Covariance update: } & \mathbf{P}[t]=\mathbf{P}[t-1]-\mathbf{K}[t] \boldsymbol{\phi}[t]^{T} \mathbf{P}[t-1] .
\end{aligned}
$$

By recursively applying (13) to the data record $\mathbf{x}[t]$ ( $t=$ $1, \ldots, L)$, the final parameter matrix estimate can be obtained as $\widehat{\boldsymbol{\theta}}[L]^{T}$, where $L$ is the length of the data record. For the initialization of the method it is customary to set $\hat{\boldsymbol{\theta}}[0]^{T}=\mathbf{0}$, $\mathbf{P}[0]=\gamma^{-1} \mathbf{I}_{k\left(n_{a} p_{a}+n_{c} p_{c}\right)}$. It should be further stressed that the recursive scheme may yield biased parameter estimates even if parameters can be described exactly as weighted sums of basis functions, as the regression matrix $\phi[t]$ contains entries that are constructed from data using past models.

Generally, the capability of the RPLR-FS-TARMA method is limited due to the following four reasons. Firstly, the estimate $\hat{\boldsymbol{\theta}}[t]^{T}$ is recursively updated at each time step, but the final estimate $\widehat{\boldsymbol{\theta}}[L]^{T}$ involves processing the entire data record. Hence, the RPLR-FS-TARMA method is a recursive, but not a real-time, time-varying system identification method. Secondly, the RPLR-FS-TARMA method, as a member of running-basis estimators [10], will encounter numerical problems in case that the basis sequences are not bounded. Hence, this method may be limited to the cases in which $L$ is known and the basis functions can be scaled in advance. Thirdly, we have to consider the problem of data saturation [23], which means we may reach a point in our analysis of data that sampling more data will not lead to more accurate estimation of $\widehat{\boldsymbol{\theta}}[L]^{T}$. Hence, this method may be limited to the cases in which $L$ is not so large or data saturation can be avoided. Finally, with the increase in $L$, the difficulty of expanding the time-dependent model parameters onto specific functional subspaces increases as well. For example, the selection of a suitable functional subspace could be very difficult, and the subspace dimensionalities $p_{a}$ and/or $p_{c}$ could be quite large. Hence, the superior achievable accuracy, lower computational complexity, and enhanced tracking capability of the RPLR-FS-TARMA method will not be guaranteed any more.

3.2. Exponentially Weighted RPLR-FS-TARMA Method. In order to avoid some of those limitations of the RPLRFS-TARMA method, the exponentially weighted RPLR-FSTARMA method is presented by including an exponentially weighted mechanism in the RPLR-FS-TARMA method $[9,11$, 13].

Given the forgetting factor $0<\lambda \leq 1$ (a positive scalar, usually close to one) and the regularization parameter $\gamma \geq$ 0 , the exponentially weighted regularized least squares cost function is defined as

$$
\min _{\boldsymbol{\theta}[t]}\left[\frac{1}{2} \sum_{\tau=1}^{t} \lambda^{t-\tau}\left\|\mathbf{x}[\tau]-\boldsymbol{\theta}[t]^{T} \boldsymbol{\phi}[\tau]\right\|^{2}+\frac{1}{2} \lambda^{t} \gamma\|\boldsymbol{\theta}[t]\|^{2}\right] .
$$

The exponentially weighted RPLR-FS-TARMA method can be obtained by minimizing the cost function of (14) and is summarized in the following $[9,11,13]$ :

$$
\begin{aligned}
\text { Estimator update: } & \widehat{\boldsymbol{\theta}}[t]^{T}=\widehat{\boldsymbol{\theta}}[t-1]^{T}+\widehat{\mathbf{e}}[t \mid t-1] \mathbf{K}[t]^{T}, \\
\text { Prediction error: } & \widehat{\mathbf{e}}[t \mid t-1]=\mathbf{x}[t]-\widehat{\mathbf{x}}[t \mid t-1]=\mathbf{x}[t]-\widehat{\boldsymbol{\theta}}[t-1]^{T} \boldsymbol{\phi}[t], \\
\text { Gain: } & \mathbf{K}[t]=\mathbf{P}[t-1] \boldsymbol{\phi}[t]\left(\lambda+\boldsymbol{\phi}[t]^{T} \mathbf{P}[t-1] \boldsymbol{\phi}[t]\right)^{-1}, \\
\text { Covariance update: } & \mathbf{P}[t]=\lambda^{-1}\left(\mathbf{P}[t-1]-\mathbf{K}[t] \boldsymbol{\phi}[t]^{T} \mathbf{P}[t-1]\right) .
\end{aligned}
$$

By recursively applying (15) to the data record $\mathbf{x}[t](t=1, \ldots, L)$, the parameter matrix estimate at each time step can be obtained as $\widehat{\boldsymbol{\theta}}[t]^{T}$. Similarly, for the initialization of the method it is customary to set $\widehat{\boldsymbol{\theta}}[0]^{T}=\mathbf{0}, \mathbf{P}[0]=$ $\gamma^{-1} \mathbf{I}_{k\left(n_{a} p_{a}+n_{c} p_{c}\right)}$. Obviously, the exponentially weighted RPLRFS-TARMA method reduces to the RPLR-FS-TARMA method of (13), when $\lambda=1$. Besides, the exponentially weighted RPLR-FS-TARMA method reduces to the exponentially weighted RPLR-TARMA method [9, 13], when $p_{a}=p_{c}=1, G_{b_{a}(1)}[t]=G_{b_{c}(1)}[t]=1$.

Some limitations of the RPLR-FS-TARMA method are relaxed by using the exponentially weighted mechanism.
Firstly, the estimate $\widehat{\boldsymbol{\theta}}[t]^{T}$ is recursively estimated at each time step, allowing the exponentially weighted RPLR-FSTARMA method to operate in real-time. Secondly, even if $L$ is unknown, the exponentially weighted RPLR-FS-TARMA method can be still implemented by selecting a suitable functional subspace with bounded basis sequences (which is the case when a trigonometric basis is used). Thirdly, the problem of data saturation is successfully overcome by putting more emphasis on recent data and deemphasizing data from remote past.

Unfortunately, the exponentially weighted RPLR-FSTARMA method may be an impractical method due to its 
fatal drawback. The FS-TARMA model structure calls for a constant parameter matrix. Therefore, if the estimate $\widehat{\boldsymbol{\theta}}[t]^{T}$ is used as the projection coefficients of the time-dependent AR and MA parameter matrices of (6) at each time step, the structured parameter evolution will be partially violated. In other words, the exponentially weighted RPLR-FS-TARMA method loses those advantages of the DPE methods and sometimes even underperforms its UPE counterpart, that is, the exponentially weighted RPLR-TARMA method $[9,13]$.

\section{Kernel Ridge Regression FS-TARMA Approach}

In the previous section we have reviewed the RPLR-FSTARMA approach which suffers from many limitations. This section proposes a kernel ridge regression FS-TARMA approach which can outperform the RPLR-FS-TARMA approach in terms of achievable accuracy and tracking capability.

4.1. Parameter Matrix Estimation. The FS-TARMA model may suffer from limited expressiveness, while this problem can be overcome by using kernel methods [24-26]. The main idea of kernel methods can be summarized as follows: project the inputs $\phi[t]$ from the input space $\mathbb{U}$ into a highdimensional feature space $\varphi(\phi[t]) \in \mathbb{W}$ via a reproducing kernel and then apply the linear model in this feature space instead of directly on the inputs themselves. Based on this idea, we propose to rewrite the FS-TARMA model of (7) into the following form:

$$
\begin{aligned}
& \mathbf{x}[t]=\boldsymbol{\theta}^{T} \boldsymbol{\phi}[t]+\mathbf{e}[t] \Longrightarrow \\
& \mathbf{x}[t]=\boldsymbol{\vartheta}^{T} \boldsymbol{\Theta}[t]+\mathbf{e}[t]
\end{aligned}
$$

with $\mathfrak{\vartheta} \in \mathbb{R}^{\mathscr{D} \times k}$ being the time-invariant parameter matrix and $\Theta[t] \triangleq \varphi(\phi[t]) \in \mathbb{R}^{\mathscr{D} \times 1}$ the corresponding regression vector. $\mathscr{D}$ denotes the dimensionality of the feature space.

Given the regularization parameter $\gamma \geq 0$, the regularized least squares cost function is defined as

$$
\min _{\boldsymbol{\vartheta}}\left[\frac{1}{2} \sum_{\tau=1}^{t}\left\|\mathbf{x}[\tau]-\boldsymbol{\vartheta}^{T} \boldsymbol{\Theta}[\tau]\right\|^{2}+\frac{1}{2} \gamma\|\boldsymbol{\vartheta}\|^{2}\right] .
$$

By using kernel ridge regression $[27,28]$, the solution to this problem is given by

$$
\widehat{\boldsymbol{\vartheta}}[t]=\left(\gamma \mathbf{I}_{D}+\widetilde{\boldsymbol{\Theta}}[t] \widetilde{\boldsymbol{\Theta}}[t]^{T}\right)^{-1} \widetilde{\boldsymbol{\Theta}}[t] \widetilde{\mathbf{x}}[t]
$$

with $\widetilde{\Theta}[t]=[\Theta[1], \Theta[2], \ldots, \Theta[t]], \widetilde{\mathbf{x}}[t]=[\mathbf{x}[1], \mathbf{x}[2], \ldots$, $\mathbf{x}[t]]^{T}$.

As the dimensionality of the feature space $\mathscr{D}$ can be very high (even infinite), (18) may be not computable in practice. By using the matrix inversion lemma $[9,26]$

$$
(\mathbf{A}+\mathbf{B C D})^{-1}=\mathbf{A}^{-1}-\mathbf{A}^{-1} \mathbf{B}\left(\mathbf{C}^{-1}+\mathbf{D A}^{-1} \mathbf{B}\right)^{-1} \mathbf{D A}^{-1}
$$

with the identifications $\gamma \mathbf{I}_{\mathscr{D}} \rightarrow \mathbf{A}, \widetilde{\Theta}[t] \rightarrow \mathbf{B}, \mathbf{I}_{t} \rightarrow \mathbf{C}$, and $\widetilde{\Theta}[t]^{T} \rightarrow \mathbf{D}$, we can rewrite (18) into the following form:

$$
\widehat{\boldsymbol{\vartheta}}[t]=\widetilde{\boldsymbol{\Theta}}[t]\left(\gamma \mathbf{I}_{t}+\widetilde{\boldsymbol{\Theta}}[t]^{T} \widetilde{\boldsymbol{\Theta}}[t]\right)^{-1} \widetilde{\mathbf{x}}[t],
$$

where $\widetilde{\boldsymbol{\Theta}}[t]^{T} \widetilde{\boldsymbol{\Theta}}[t]$ is computable by using the "kernel trick" $\kappa(\phi[i], \phi[j])=\boldsymbol{\Theta}[i]^{T} \boldsymbol{\Theta}[j][24-28]$, as follows:

$$
\widetilde{\Theta}[t]^{T} \widetilde{\Theta}[t]=\left[\begin{array}{ccc}
\kappa(\phi[1], \phi[1]) & \cdots & \kappa(\phi[1], \phi[t]) \\
\vdots & \ddots & \vdots \\
\kappa(\phi[t], \phi[1]) & \cdots & \kappa(\phi[t], \phi[t])
\end{array}\right]
$$

with $\kappa(\cdot, \cdot)$ is called a reproducing kernel of $\mathbb{\square}[29]$, and its form can be finalized after the selection of kernel functions.

Denoting $\mathbf{Q}[t]=\gamma \mathbf{I}_{t}+\widetilde{\boldsymbol{\Theta}}[t]^{T} \widetilde{\boldsymbol{\Theta}}[t]$ and $\mathbf{k}[t]=\widetilde{\boldsymbol{\Theta}}[t-$ $1]^{T} \boldsymbol{\Theta}[t]$, we have

$$
\mathbf{Q}[t]=\left[\begin{array}{cc}
\mathbf{Q}[t-1] & \mathbf{k}[t] \\
\mathbf{k}[t]^{T} & \gamma+\kappa(\phi[t], \phi[t])
\end{array}\right] .
$$

Obviously, the dimensionality of $\mathbf{Q}[t]$ increases as the number of samples increases. If the dimensionality of $\mathbf{Q}[t]$ is smaller than a predefined sliding-window length $N$, by using the block matrix inversion identity [26]

$$
\begin{aligned}
& {\left[\begin{array}{ll}
\mathbf{A} & \mathbf{B} \\
\mathbf{C} & \mathbf{D}
\end{array}\right]^{-1}} \\
& =\left[\begin{array}{cc}
\left(\mathbf{A}-\mathbf{B D}^{-1} \mathbf{C}\right)^{-1} & -\mathbf{A}^{-1} \mathbf{B}\left(\mathbf{D}-\mathbf{C A}^{-1} \mathbf{B}\right)^{-1} \\
-\mathbf{D}^{-1} \mathbf{C}\left(\mathbf{A}-\mathbf{B D}^{-1} \mathbf{C}\right)^{-1} & \left(\mathbf{D}-\mathbf{C A}^{-1} \mathbf{B}\right)^{-1}
\end{array}\right]
\end{aligned}
$$

the inverse matrix of $\mathbf{Q}[t]$ can be computed by

$$
\begin{aligned}
\mathbf{Q} & {[t]^{-1} } \\
& =\left[\begin{array}{cc}
\mathbf{Q}[t-1]^{-1}+\mathbf{z}[t] \mathbf{z}[t]^{T} r[t]^{-1} & -\mathbf{z}[t] r[t]^{-1} \\
-\mathbf{z}[t]^{T} r[t]^{-1} & r[t]^{-1}
\end{array}\right]
\end{aligned}
$$

with $\mathbf{z}[t]=\mathbf{Q}[t-1]^{-1} \mathbf{k}[t]$ and $r[t]=\gamma+\kappa(\phi[t], \phi[t])-$ $\mathbf{z}[t]^{T} \mathbf{k}[t]$.

Assume that $N_{1} \geq 1$ and $N_{2} \geq 1$ denote, respectively, the rate of data forgetting and the degree of data overlap, and $N=N_{1}+N_{2}$. When the dimensionality of $\mathbf{Q}[t]$ equals the sliding-window length $N$, we need to downsize the matrix $\mathbf{Q}[t]$ and obtain the corresponding downsized matrix $\mathbf{Q}^{\#}[t] \epsilon$ $\mathbb{R}^{N_{2} \times N_{2}}$ by removing the first $N_{1}$ rows and columns of $\mathbf{Q}[t]$. The inverse matrix of $\mathbf{Q}^{\#}[t]$ can be computed by using the following downsized matrix inversion formula. Assume that the previously obtained $\mathbf{Q}[t]^{-1}$ is of the form

$$
\mathbf{Q}[t]^{-1}=\left[\begin{array}{cc}
*_{N_{1} \times N_{1}} & *_{N_{1} \times N_{2}} \\
*_{N_{2} \times N_{1}} & \mathbf{Q}^{\#}[t]
\end{array}\right]^{-1}=\left[\begin{array}{cc}
\mathbf{A} & \mathbf{B} \\
\mathbf{C} & \mathbf{D}
\end{array}\right] ;
$$

we have

$$
\mathbf{Q}^{\#}[t]^{-1}=\mathbf{D}-\mathbf{C A}^{-1} \mathbf{B} .
$$

Downsize $\widetilde{\Theta}[t] \leftarrow\left[\Theta\left[t-N_{2}+1\right], \Theta\left[t-N_{2}+2\right], \ldots, \Theta[t]\right]$, $\widetilde{\mathbf{x}}[t] \leftarrow\left[\mathbf{x}\left[t-N_{2}+1\right], \mathbf{x}\left[t-N_{2}+2\right], \ldots, \mathbf{x}[t]\right]^{T}$ and redefine 
$\mathbf{Q}[t] \leftarrow \mathbf{Q}^{\#}[t]$; we have the newly defined matrix $\mathbf{Q}[t] \epsilon$ $\mathbb{R}^{N_{2} \times N_{2}}$, whose dimensionality $N_{2}$ is smaller than $N$. Once the next data observation has been seen, $\mathbf{Q}[t+1]$ can be upsized by (22) and its inverse matrix $\mathbf{Q}[t+1]^{-1}$ can be computed by (24). In case the dimensionality of $\mathbf{Q}\left[t+N_{1}\right]$ equals $N$, first compute $\mathbf{Q}^{\#}\left[t+N_{1}\right]^{-1}$ by using the downsized matrix inversion formulas (25) and (26), and then redefine $\mathbf{Q}\left[t+N_{1}\right] \leftarrow \mathbf{Q}^{\#}\left[t+N_{1}\right]$.

Substituting $\mathbf{Q}[t]^{-1}$ into (20), the parameter matrix $\boldsymbol{9}$ at each time step can be obtained by

$$
\widehat{\boldsymbol{\vartheta}}[t]=\widetilde{\boldsymbol{\Theta}}[t] \mathbf{Q}[t]^{-1} \widetilde{\mathbf{x}}[t] .
$$

In order to avoid violating the structured parameter evolution of the FS-TARMA model, the parameter matrix $\widehat{\boldsymbol{\vartheta}}[t]$ is updated once every $N_{1}$ data observations. In other words, the parameter matrix $\widehat{\boldsymbol{\vartheta}}[t]$ is updated by (27) only when the dimensionality of $\mathbf{Q}[t]$ is equal to $N$. It should be noted that each $\widehat{\boldsymbol{\vartheta}}[t]$ is estimated based on the data observations within $[t-N+1, t]$, and each $\widehat{\vartheta}[t]$ can be subsequently used to compute the FS-TARMA model parameter matrix $\widehat{\boldsymbol{\theta}}$ within $[t-N+1, t]$. Furthermore, both of the adjacent parameter matrix estimates $\widehat{\boldsymbol{\vartheta}}[t]$ and $\widehat{\boldsymbol{\vartheta}}\left[t+N_{1}\right]$ can be used to compute $\widehat{\boldsymbol{\theta}}$ at each arbitrary time step within $\left[t-N_{2}+1, t\right]$, and that is why $\mathrm{N}_{2}$ is called the degree of data overlap.

Finally, the FS-TARMA model parameter matrix $\widehat{\boldsymbol{\theta}}$ can be obtained by substituting (27) into (16):

$$
\begin{aligned}
& \widehat{\boldsymbol{\theta}}[t]^{T}=\widehat{\boldsymbol{\vartheta}}[t]^{T} \boldsymbol{\varphi}\left(\mathbf{I}_{k\left(n_{a} p_{a}+n_{c} p_{c}\right)}\right) \\
& =\widetilde{\mathbf{x}}[t]^{T} \mathbf{Q}[t]^{-1}\left[\begin{array}{c}
\kappa\left(\phi[t-N+1], \mathbf{I}_{k\left(n_{a} p_{a}+n_{c} p_{c}\right)}\right) \\
\kappa\left(\phi[t-N+2], \mathbf{I}_{k\left(n_{a} p_{a}+n_{c} p_{c}\right)}\right) \\
\vdots \\
\kappa\left(\phi[t], \mathbf{I}_{k\left(n_{a} p_{a}+n_{c} p_{c}\right)}\right)
\end{array}\right] .
\end{aligned}
$$

4.2. Model Structure Selection. The model structure selection for the KRR-FS-TARMA method includes the selection of basis functions (a suitable functional subspace with proper dimensionalities $p_{a}, p_{c}$ and indices $\left.b_{a}(i), b_{c}(i)\right)$, the kernel function $\kappa(\cdot, \cdot)$, the regularization parameter $\gamma$, the slidingwindow length $N$ and the data forgetting rate $N_{1}$, and the AR and MA orders $n_{a}$ and $n_{c}$. The Akaike information criterion and the Bayesian information criterion may not be formally used in connection with methods that recursively estimate the AR and MA parameter matrices at each time step [11]. Therefore, once the functional subspaces, $p_{a}, p_{c}, b_{a}(i), b_{c}(i)$, $\kappa(\cdot, \cdot), \gamma, N$, and $N_{1}$, have been initially selected, model orders including AR and MA orders will be selected based on the residual sum of squares (RSS) criterion $[9,11-13]$ as

$$
\mathrm{RSS}=\sum_{\tau=1}^{L} \widehat{\mathbf{e}}[\tau]^{T} \cdot \widehat{\mathbf{e}}[\tau]
$$

with $\widehat{\mathbf{e}}[\tau]$ being the estimated innovations (residual) sequence vector.
Model structure selection may be viewed as an iterative optimization problem that can be tackled via following three steps.

Step 1. Select a suitable functional subspace with proper dimensionalities $p_{a}, p_{c}$ and indices $b_{a}(i), b_{c}(i)$, a suitable kernel function $\kappa(\cdot, \cdot)$, the initial values of $\gamma, N$ and $N_{1}$ based upon prior knowledge, physical understanding, and expected form of model parameter variation.

Step 2. Select model orders including AR and MA orders $n_{a}$ and $n_{c}$ via optimization procedure [14] based upon the minimization of the RSS used as "fitness" function:

(a) AR and MA orders $\left(n_{a}=n_{c}\right)$ within the search space are sequentially selected, and the best fit AR order $\widehat{n}_{a}$ can be determined when the decrease of RSS values is quite subtle.

(b) MA orders within the search space $\left[0, \widehat{n}_{a}\right]$ are sequentially selected under the previously obtained AR order $\widehat{n}_{a}$, and the best fit MA order $\widehat{n}_{c}$ can be determined in the same way as that of AR order.

Step 3. Refine the candidate model structure obtained in Step 2 by further optimizing those parameters including $p_{a}$, $p_{c}, b_{a}(i), b_{c}(i), \gamma, N$, and $N_{1}$.

The workflow of the KRR-FS-TARMA approach is summarized as follows:

Model structure selection: basis functions including $p_{a}, p_{c}, b_{a}(i)$, and $b_{c}(i)$, kernel function $\kappa(\cdot, \cdot)$, AR and MA orders $n_{a}$ and $n_{c}$, regularization parameter $\gamma$, sliding-window length $N$, forgetting rate $N_{1}$, and overlap degree $N_{2}=N-N_{1}$ Initialization $(t=1)$ :

$$
\begin{aligned}
\mathbf{Q}[1]^{-1} & =(\gamma+\kappa(\phi[1], \phi[1]))^{-1}, \\
\widetilde{\boldsymbol{\Theta}}[1] & =\boldsymbol{\Theta}[1], \\
\widetilde{\mathbf{x}}[1] & =\mathbf{x}[1]^{T}
\end{aligned}
$$

Computation $(t>1)$ :

$$
\text { if } \operatorname{dim}(\mathbf{Q}[t])<N
$$

Upsizing: $\widetilde{\Theta}[t] \leftarrow[\widetilde{\Theta}[t-1], \Theta[t]], \widetilde{\mathbf{x}}[t] \leftarrow$ $\left[\widetilde{\mathbf{x}}[t-1] ; \mathbf{x}[t]^{T}\right]$

Upsizing: obtain $\mathbf{Q}[t]$ according to Eq. (22)

Compute $\mathbf{Q}[t]^{-1}$ according to Eq. (24)

elseif $\operatorname{dim}(\mathbf{Q}[t])=N$

Compute the FS-TARMA model parameter matrix $\widehat{\boldsymbol{\theta}}[t]^{T}$ according to Eq. (28)

Compute the time-dependent AR parameter matrix $\widehat{\mathbf{A}}_{i}[t]$ according to Eq. (6) Compute the modal parameters according to Eq. (2), (3) and (4) 


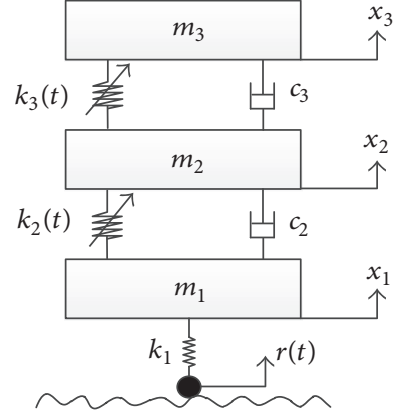

Figure 1: A time-varying structural system.

TABLE 1: Time-varying system parameters.

\begin{tabular}{ll}
\hline Property & Value \\
\hline Mass $(\mathrm{kg})$ & $m_{1}=0.5, m_{2}=1.5, m_{3}=1.0$ \\
\hline $\begin{array}{l}\text { Damping } \\
(\mathrm{N} /(\mathrm{m} / \mathrm{s}))\end{array}$ & $c_{2}=0.5, c_{3}=0.3$ \\
\hline & $k_{1}=300$ \\
Stiffness $(\mathrm{N} / \mathrm{m})$ & $k_{2}(t)=100+60 \sin \left(\frac{2 \pi t}{170}\right)+20 \sin \left(\frac{2 \pi t}{85}\right)$ \\
& $k_{3}(t)=120+72 \sin \left(\frac{2 \pi t}{141.67}\right)+24 \sin \left(\frac{2 \pi t}{94.44}\right)$ \\
\hline
\end{tabular}

Downsizing: obtain $\mathbf{Q}^{\#}[t]$ by removing the first $N_{1}$ rows and columns of $\mathbf{Q}[t]$

Compute $\mathbf{Q}^{\#}[t]^{-1}$ according to Eq. (25) and

(26)

Downsizing:

$$
\begin{gathered}
\widetilde{\boldsymbol{\Theta}}[t] \longleftarrow\left[\boldsymbol{\Theta}\left[t-N_{2}+1\right], \boldsymbol{\Theta}\left[t-N_{2}+2\right], \ldots, \boldsymbol{\Theta}[t]\right] \\
\widetilde{\mathbf{x}}[t] \longleftarrow\left[\mathbf{x}\left[t-N_{2}+1\right], \mathbf{x}\left[t-N_{2}+2\right], \ldots, \mathbf{x}[t]\right]^{T} \\
\text { Redefining: } \mathbf{Q}[t] \leftarrow \mathbf{Q}^{\#}[t] \\
\text { end if }
\end{gathered}
$$

\section{Numerical Validation}

5.1. The Time-Varying Structural System. For validating the proposed method for output-only modal parameter recursive estimation of time-varying structures, the three-degree-offreedom structural system given by $[11,30]$ is used in this section, as shown in Figure 1. This system represents a simplified model of one-quarter of a truck vehicle [11,30], which is subject to a unobservable, stationary, Gaussian, zero mean, and uncorrelated road excitation $\mathbf{r}(t)$. The numerical values of system parameters are given in Table 1.

Given a particular excitation realization, the vibration displacement responses for $t \in[-16.67,850] \mathrm{s}$ are obtained via the Runge-Kutta 4/5 method characterized by variable integration step and subsequently recorded at a sampling frequency $12 \mathrm{~Hz}[11,30]$. The initial 200 samples of the obtained responses for $t \in[-16.67,0] \mathrm{s}$ are discarded to minimize initialization effects. The rest of the obtained responses for $t \in[0,850] \mathrm{s}$ are corrupted by Gaussian white noise with the signal-to-noise ratio of $20 \mathrm{~dB}$. In a word, each noise-corrupted response realization, sampled at $12 \mathrm{~Hz}$, is 850 seconds long, producing 10201 sample-long versions of the nonstationary vibration signals. This procedure is repeated for each Monte Carlo run (number of runs: 30), with a different excitation realization used each time.

5.2. Modal Parameter Estimation Results. The proposed KRRFS-TARMA method is, along with the existing RPLR-FSTARMA method, applied to estimate modal parameters of the time-varying numerical system. The proposed method is assessed against the theoretical modal parameters, and further comparisons with the RPLR-FS-TARMA method are also made via Monte Carlo experiments.

5.2.1. The RPLR-FS-TARMA Method Based Estimation. The functional basis spanned by trigonometric functions of the form $[11,30]$

$$
\begin{aligned}
G_{0}[t] & =1, \\
G_{2 k-1}[t] & =\sin \left[\frac{k \pi(t-1)}{L-1}\right], \\
G_{2 k}[t] & =\cos \left[\frac{k \pi(t-1)}{L-1}\right],
\end{aligned}
$$

$$
k=1,2, \ldots,
$$

is selected. The corresponding dimensionalities and indices are, respectively, $p_{a}=40, b_{a}=\left[\begin{array}{lll}0 & : 39\end{array}\right]$, and $p_{c}=6$, $b_{c}=[0: 5]$. The value of $\gamma=10^{-4}$ is selected. Model order selection is achieved via the RSS criterion, as the RPLR-FSTARMA method is based on the class of recursive TARMA representations. Figure 2(a) depicts the RSS normalized by the sum of squares of the signal samples (series sum of squares, SSS) of the obtained RPLR-FS-TARMA $(p, q)(p=$ $q=1,2,3,4)$, which suggests AR order $n_{a}=2$. Figure 2(b) depicts the RSS normalized by the SSS of the obtained RPLRTARMA $(p, q)(p=2, q=0,1,2)$, which suggests MA order $n_{c}=2$. Figure 3 depicts the "frozen-time" RPLR-FSTARMA based modal parameter estimates (with damping ratio $0<\zeta_{i}[t]<0.1$ ) from the 30 Monte Carlo tests, along with their theoretical counterparts. The blue dots notate the modal parameter estimates from the 30 tests, and the green dashed lines notate the theoretical modal parameters.

\subsubsection{The Exponentially Weighted RPLR-TARMA Method}

Based Estimation. The functional basis given by (32) is selected with $p_{a}=p_{c}=1, b_{a}=b_{c}=0$. In other words, the exponentially weighted RPLR-FS-TARMA method reduces to its UPE counterpart, that is, the exponentially weighted RPLR-TARMA method. The values of $\gamma=10^{-4}$ and $\lambda=$ 0.988 are selected. Figure 4(a) depicts the RSS normalized by the SSS of the obtained RPLR-TARMA $(p, q)(p=q=$ $1,2,3,4)$, which suggests AR order $n_{a}=2$. Figure 4(b) depicts the RSS normalized by the SSS of the obtained RPLRTARMA $(p, q)(p=2, q=0,1,2)$, which suggests MA order $n_{c}=2$. Figure 5 depicts the "frozen-time" RPLR-TARMA based modal parameter estimates (with damping ratio $0<$ 


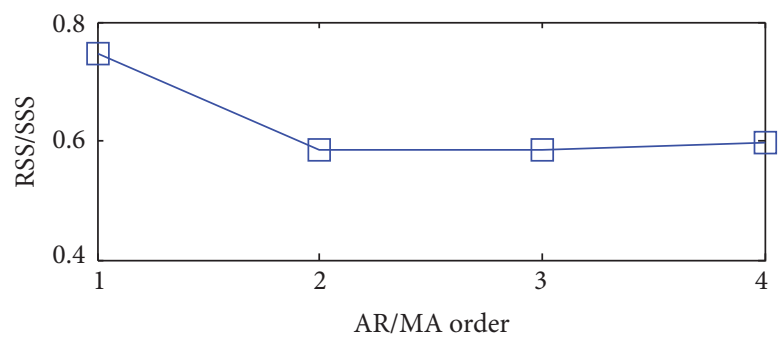

(a)

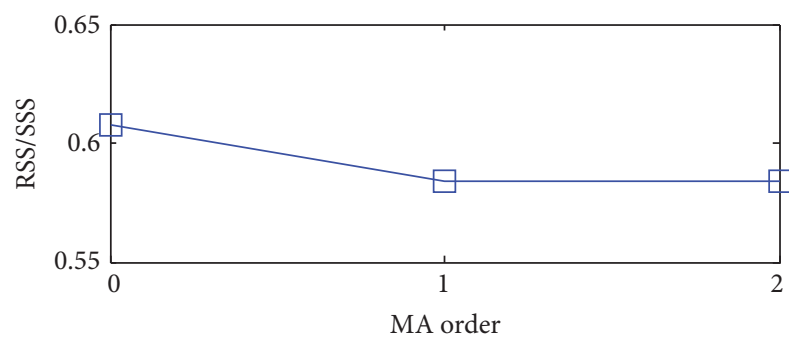

(b)

FIGURE 2: RPLR-FS-TARMA model order selection: (a) AR order and (b) MA order.

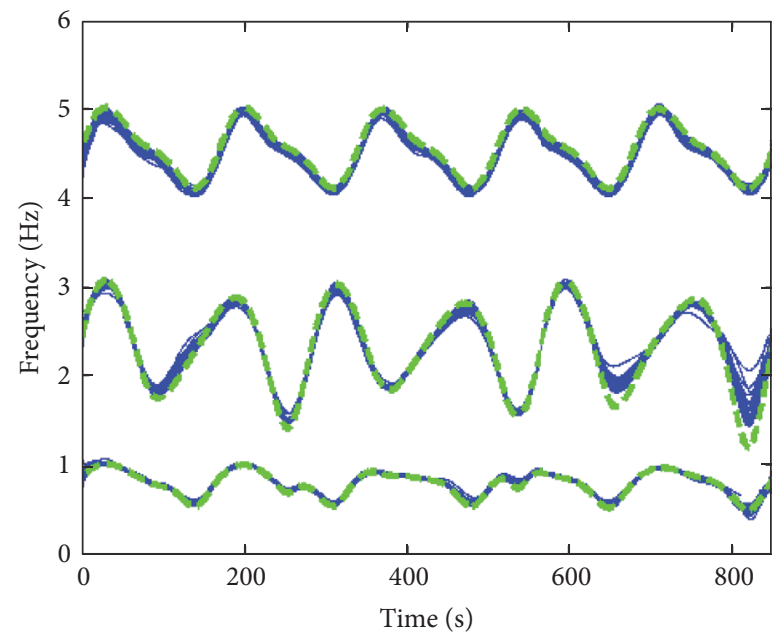

(a)
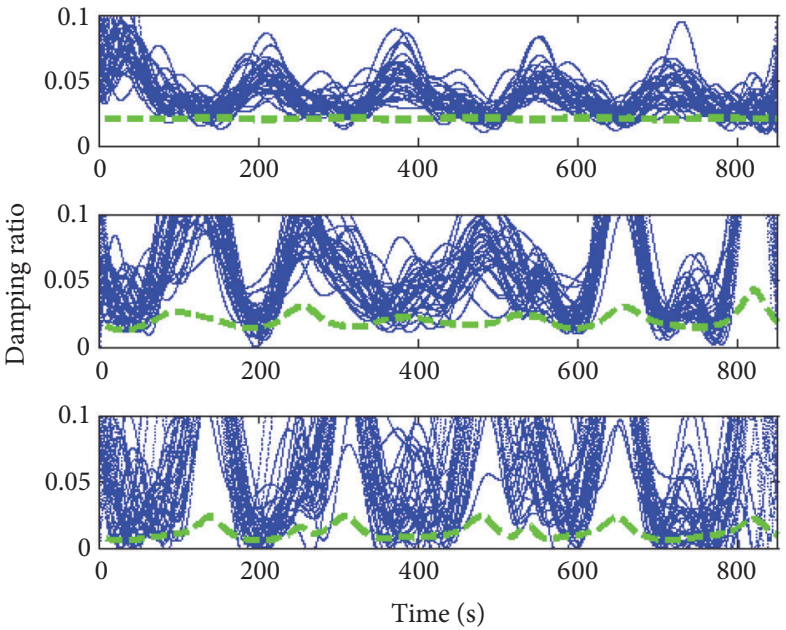

(b)

FIGURE 3: RPLR-FS-TARMA based modal parameter Monte Carlo estimates (30 realizations): (a) natural frequency estimates and (b) damping ratio estimates.

$\left.\zeta_{i}[t]<0.1\right)$ from the 30 Monte Carlo tests, along with their theoretical counterparts.

5.2.3. The KRR-FS-TARMA Method Based Estimation. The functional basis given by (32) is selected with $p_{a}=20, b_{a}=$ [0:19] and $p_{c}=3, b_{c}=[0: 2]$. The simplest linear kernel $\kappa(\phi[i], \phi[j])=\phi[i]^{T} \phi[j]$ is here selected. The values of $\gamma=$ $10^{-3}, N=1020$, and $N_{1}=510$ are selected. Figure 6(a) depicts the RSS normalized by the SSS of the obtained KRRFS-TARMA $(p, q)(p=q=1,2,3,4)$, which suggests AR order $n_{a}=2$. Figure $6(\mathrm{~b})$ depicts the RSS normalized by the SSS of the obtained KRR-FS-TARMA $(p, q)(p=2, q=$ $0,1,2)$, which suggests MA order $n_{c}=2$. Figure 7 depicts the "frozen-time" KRR-FS-TARMA based modal parameter estimates (with damping ratio $0<\zeta_{i}[t]<0.1$ ) from the 30 Monte Carlo tests, along with their theoretical counterparts.

5.2.4. Comparisons. From the natural frequency estimates shown in Figures 3 and 5 it may be observed that the RPLRFS-TARMA based natural frequency estimates exhibit better accuracy and tracking than its UPE counterpart obtained by the exponentially weighted RPLR-TARMA method. From the natural frequency estimates shown in Figures 3, 5, and 7 it may be further observed that the KRR-FS-TARMA based natural frequency estimates exhibit best accuracy and tracking than its RPLR-FS-TARMA and exponentially weighted RPLR-TARMA counterparts. The damping ratios are filtered by $0<\zeta_{i}[t]<0.1$, and their estimates are generally much less accurate, which is common for almost all methods.

In order to better compare these methods by using Monte Carlo natural frequency estimates, a mean absolute error (MAE) is introduced as

$$
\operatorname{MAE}=\frac{1}{R} \sum_{i=1}^{R}\left(\frac{1}{L} \sum_{t=1}^{L}\left|f(t)-\widehat{f}_{i}(t)\right|\right)
$$

with $f(t)$ designating the baseline value of the natural frequency of interest, $\hat{f}_{i}(t)$ its estimated value based on the $i$ th Monte Carlo run, $L$ the length of samples, and $R$ the number of Monte Carlo runs. Figure 8 illustrates the MAE values obtained by each method. Evidently, the identified KRR-FSTARMA model attains best overall performance compared to its RPLR-FS-TARMA and exponentially weighted RPLRTARMA counterparts.

\section{Experimental Validation}

6.1. The Laboratory Time-Varying Structure. A coupled moving mass and simply supported beam time-varying structure 


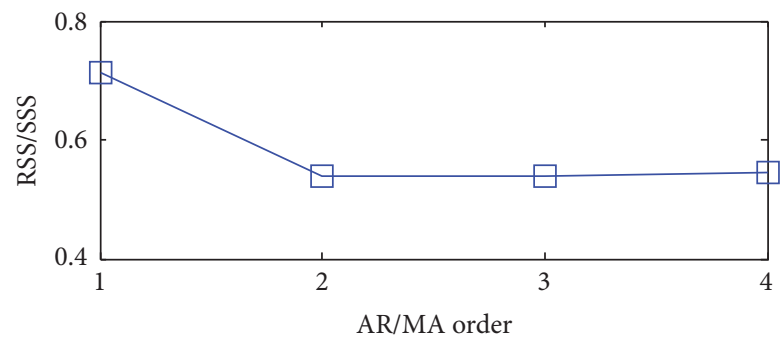

(a)

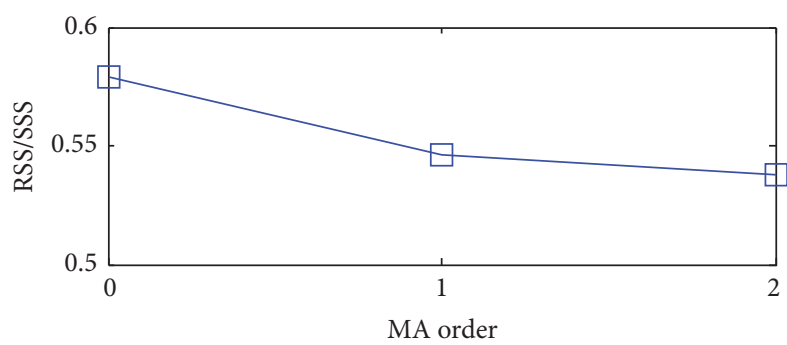

(b)

FIGURE 4: Exponentially weighted RPLR-TARMA model order selection: (a) AR order and (b) MA order.

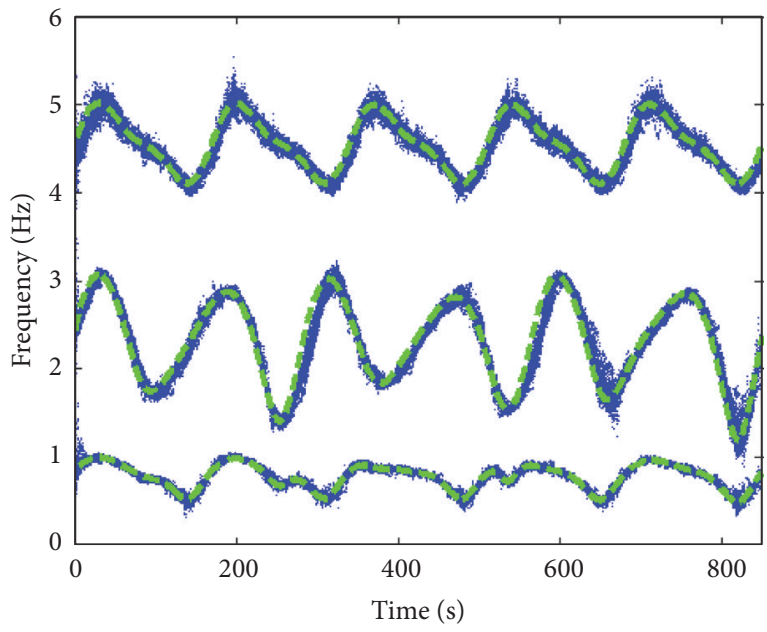

(a)
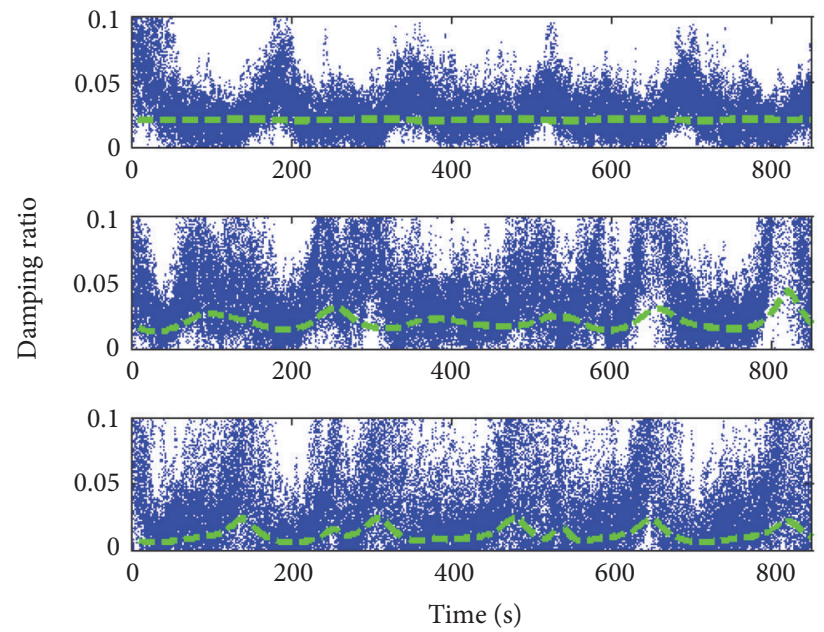

(b)

FIGURE 5: Exponentially weighted RPLR-TARMA based modal parameter Monte Carlo estimates (30 realizations): (a) natural frequency estimates and (b) damping ratio estimates.

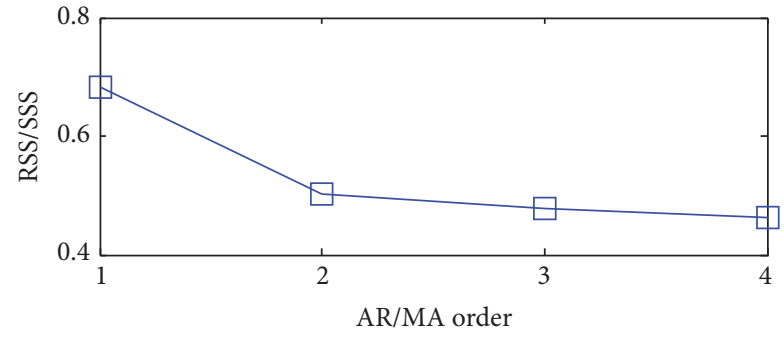

(a)

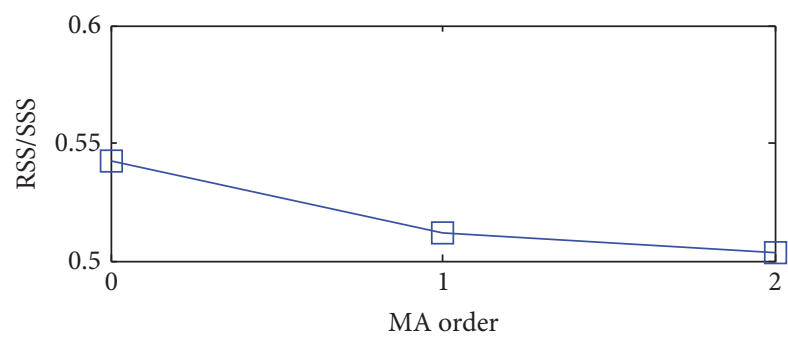

(b)

FIGURE 6: KRR-FS-TARMA model order selection: (a) AR order and (b) MA order.

[31] is used for experimental validation of the proposed KRRFS-TARMA approach. The experimental system is composed of the test structure, an exciter system, force and motion transducers, measurement and analysis systems, control systems, and boundary conditions. Figure 9 shows the schematic diagram of the experimental system and its set-up.

A nonstationary experiment is conducted with the moving mass sliding on the beam with a uniform velocity of $0.2 \mathrm{~m} / \mathrm{s}$. Figure 10(a) shows its motion form, that is, the position over time. In the experiment, no external excitation is generated to excite the coupled time-varying system (the shaker shown in Figure 9 is not in use), and the system is only excited by the motion of the moving mass. Fifteen accelerometers are used to measure the acceleration responses of the simply supported beam at fifteen uniformly distributed positions along the axial direction of the beam from left to right, as shown in Figure 9. The vertical acceleration signals, sampled at $256 \mathrm{~Hz}$, are 32 seconds long, producing 8192 sample-long versions of the nonstationary vibration signals. Figure 10(b) shows the first four baseline natural frequencies of the coupled time-varying system, obtained by using the "frozen-time" technique [31]. 


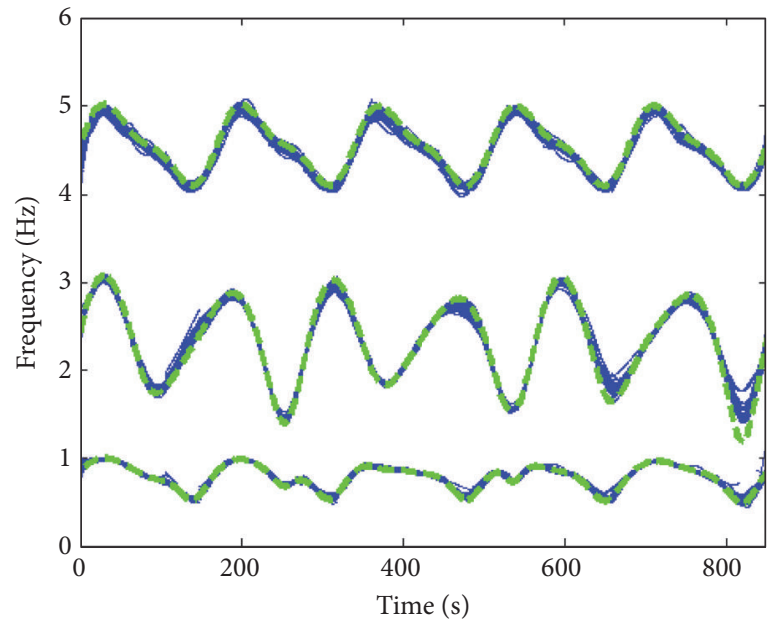

(a)
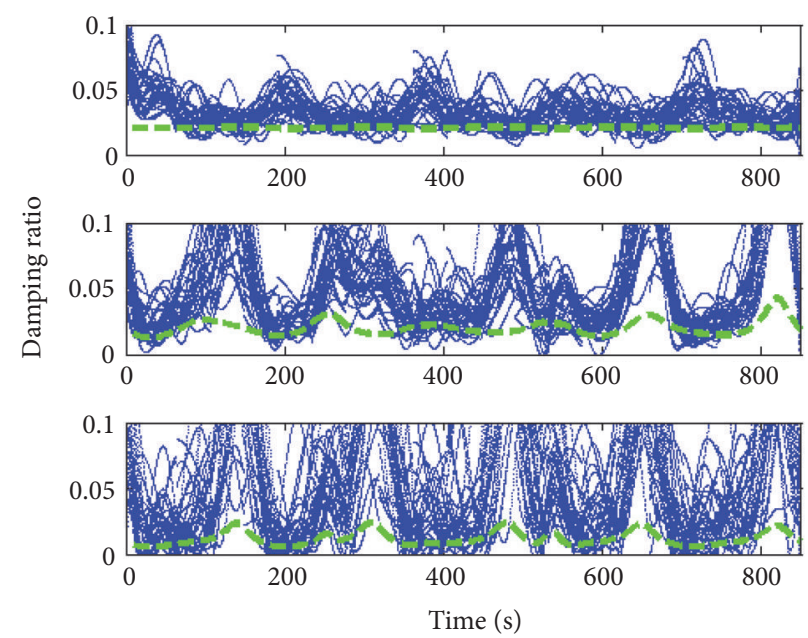

(b)

FIGURE 7: KRR-FS-TARMA based modal parameter Monte Carlo estimates (30 realizations): (a) natural frequency estimates and (b) damping ratio estimates.

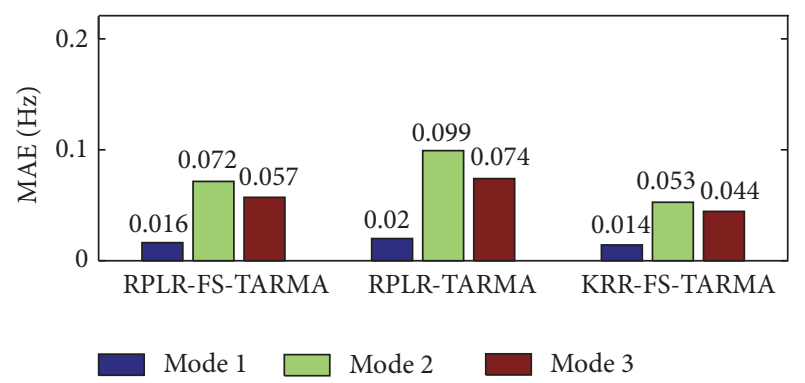

FIGURE 8: Monte Carlo comparison of different methods: MAE of natural frequency estimates.

6.2. Modal Parameter Estimation Results. In order to effectively and reliably assess and compare various methods, the study in this section is also carried out based upon Monte Carlo runs (number of runs: 30 ). In the same manner as previously introduced, 30 tests are repeatedly carried out, and the coupled time-varying system undergoes the same variation in each test. The modal parameter estimation of the experimental time-varying structure is subsequently considered based on three methods including the RPLR-FSTARMA method, the exponentially weighted RPLR-TARMA method, and the proposed KRR-FS-TARMA method.

6.2.1. The RPLR-FS-TARMA Method Based Estimation. The functional basis given by (32) is selected with $p_{a}=50, b_{a}=$ [0 : 49] and $p_{c}=1, b_{c}=0$. The value of $\gamma=10^{-4}$ is selected. Figure 11(a) depicts the RSS normalized by the SSS of the obtained RPLR-FS-TARMA $(p, q)(p=q=1,2,3,4)$, which suggests AR order $n_{a}=2$. Figure 11(b) depicts the RSS normalized by the SSS of the obtained RPLR-FS-TARMA $(p, q)(p=2, q=0,1,2)$, which suggests MA order $n_{c}=1$. Figure 12 depicts the "frozen-time" RPLR-FS-TARMA based modal parameter estimates (with damping ratio $0<\zeta_{i}[t]<$ 0.1 ) from the 30 Monte Carlo tests, along with their baseline counterparts.
6.2.2. The Exponentially Weighted RPLR-TARMA Method Based Estimation. The exponentially weighted RPLRTARMA method is employed for modal parameter estimation of the laboratory structure with $\gamma=10^{-4}$ and $\lambda=0.964$. Figure 13(a) depicts the RSS normalized by the SSS of the obtained $\operatorname{RPLR}-\mathrm{TARMA}(p, q)(p=q=1,2,3,4)$, which suggests $\mathrm{AR}$ order $n_{a}=2$. Figure 13(b) depicts the RSS normalized by the SSS of the obtained RPLR-TARMA ( $p$, q) $(p=2, q=0,1,2)$, which suggests MA order $n_{c}=0$. Figure 14 depicts the "frozen-time" RPLR-TARMA based modal parameter estimates (with damping ratio $0<\zeta_{i}[t]<$ 0.1 ) from the 30 Monte Carlo tests, along with their baseline counterparts.

6.2.3. The KRR-FS-TARMA Method Based Estimation. The functional basis given by (32) is selected with $p_{a}=40, b_{a}=$ [0 : 39] and $p_{c}=1, b_{c}=0$. The linear kernel functions, $\gamma=$ $10^{-4}, N=1024$, and $N_{1}=512$ are selected. Figure 15(a) depicts the RSS normalized by the SSS of the obtained KRRFS-TARMA $(p, q)(p=q=1,2,3,4)$, which suggests AR order $n_{a}=2$. Figure $6(\mathrm{~b})$ depicts the RSS normalized by

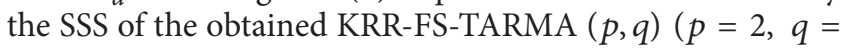
$0,1,2)$, which suggests MA order $n_{c}=2$. Figure 16 depicts the "frozen-time" KRR-FS-TARMA based modal parameter estimates (with damping ratio $0<\zeta_{i}[t]<0.1$ ) from the 30 Monte Carlo tests, along with their baseline counterparts.

6.2.4. Comparisons. From the natural frequency estimates shown in Figure 14 it may be observed that significant scatter is evidently associated with the estimated modes, and a number of false modes appear, especially in the frequency range near the third and fourth modes. Compared with the exponentially weighted RPLR-TARMA based estimates in Figure 14, the RPLR-FS-TARMA based natural frequency estimates appear more smooth and less false modes are exhibited, as shown in Figure 12. Compared with the RPLR-FS-TARMA based estimates in Figure 12, the KRR-FS-TARMA based 

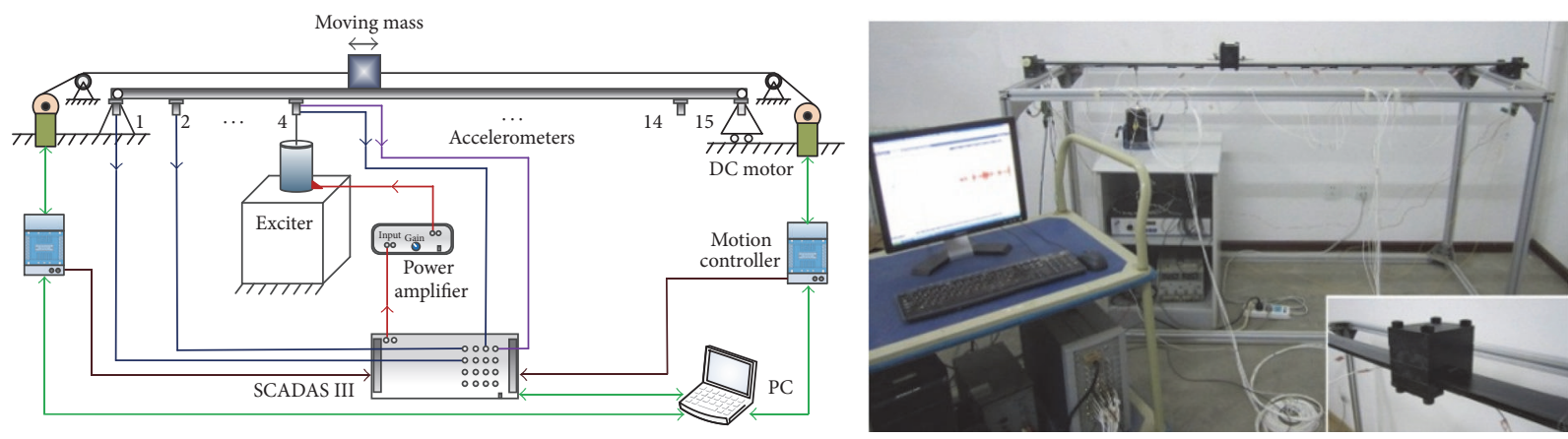

FIGURE 9: Schematic diagram of the experimental system and photo of its set-up.

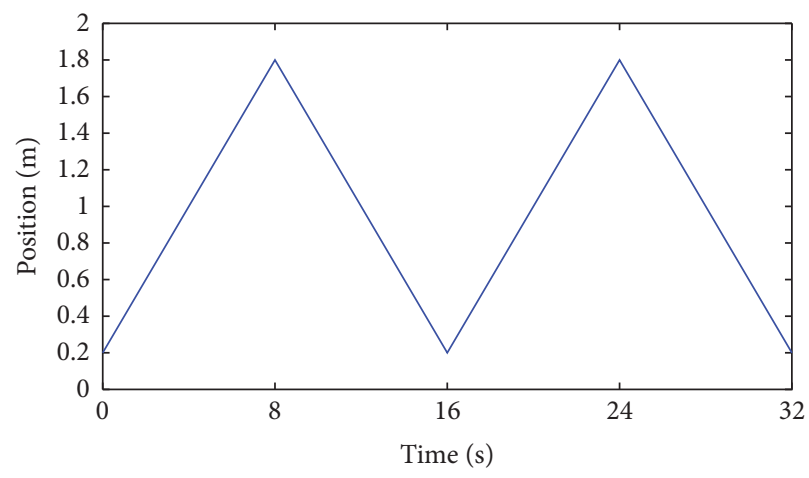

(a)

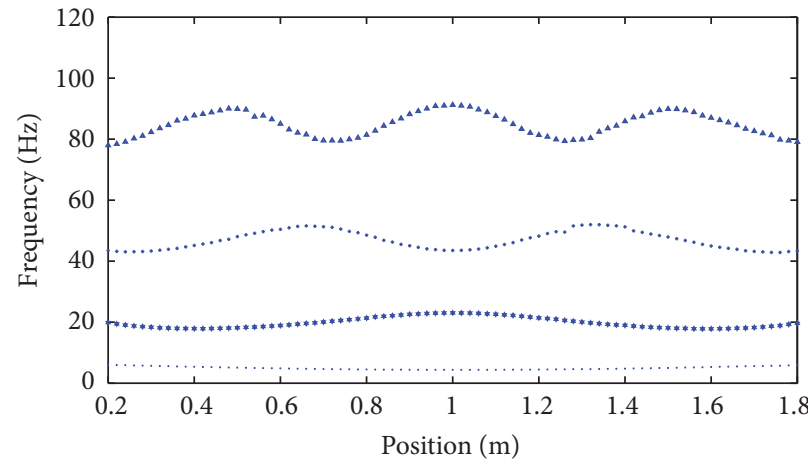

Mode $1 \cdot$ Mode $2 \cdot$ Mode $3 \cdot$ Mode 4

(b)

FIGURE 10: The motion form of the moving mass and the corresponding first four baseline natural frequencies of the experimental system: (a) motion form and (b) baseline natural frequencies.

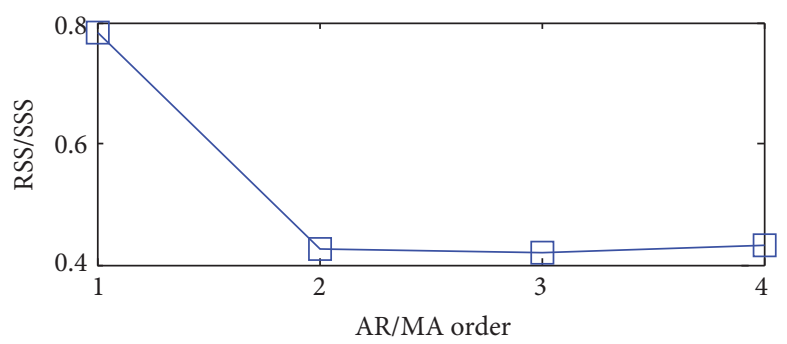

(a)

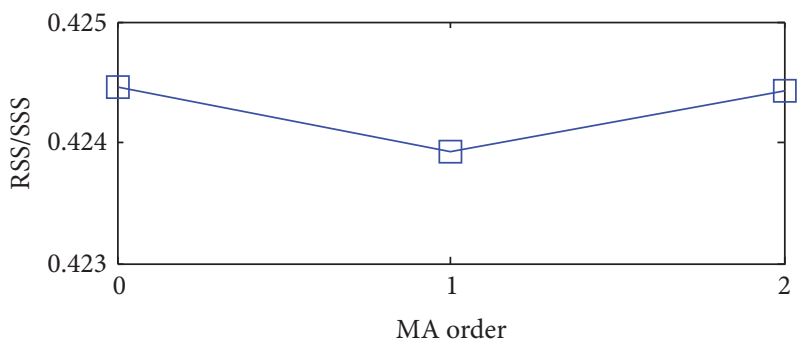

(b)

FIGURE 11: RPLR-FS-TARMA model order selection: (a) AR order and (b) MA order.

natural frequency estimates shown in Figure 16 exhibit better accuracy and tracking, especially for the third and fourth modes. The damping ratios are filtered by $0<\zeta_{i}[t]<0.1$, and their estimates are generally much less accurate. Therefore quantitative analysis is carried out based on the natural frequency estimates instead of the damping ratio estimates. Figure 17 illustrates the MAE values obtained by each method. Compared with the exponentially weighted RPLR-TARMA, its DPE counterparts including the RPLRFS-TARMA method and the KRR-FS-TARMA method can accurately track the third and fourth modes, but not for the first mode. However, the identified KRR-FS-TARMA model attains better overall performance than its RPLR-FS-TARMA counterpart.

\section{Conclusions}

This work focuses on the output-only modal parameter estimation of time-varying structures by using recursive FS-TARMA approach. A KRR-FS-TARMA approach was proposed by exclusively using the available response measurements. The proposed method was numerically and experimentally validated and further assessed against the existing RPLR-FS-TARMA method via Monte Carlo experiments. 


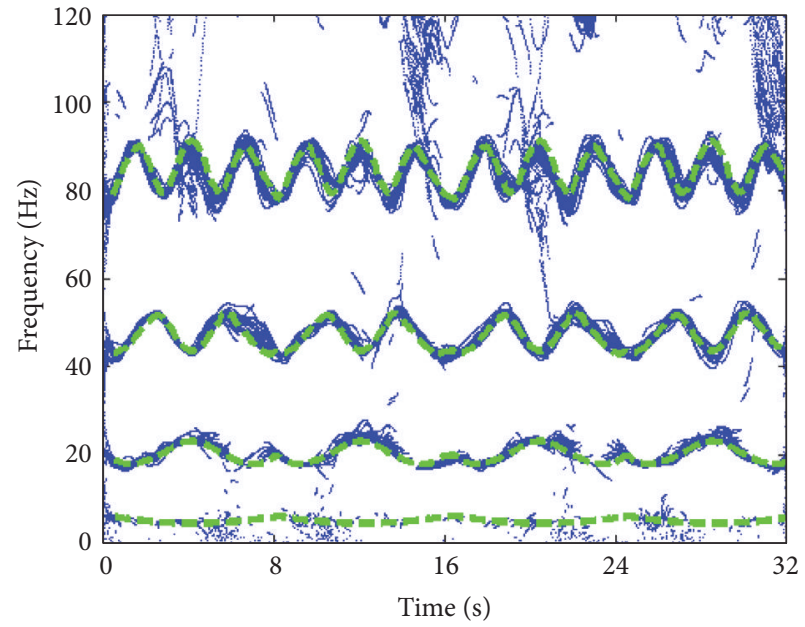

(a)
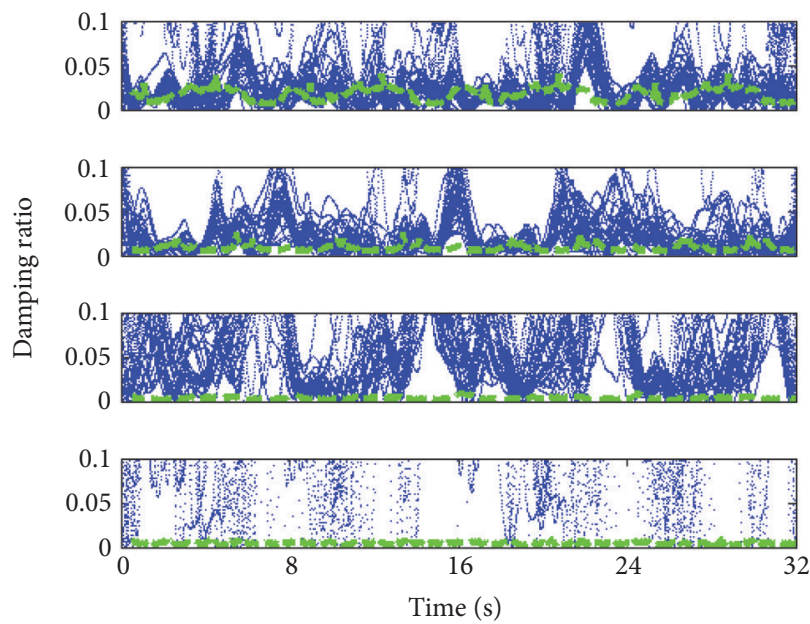

(b)

FIGURE 12: RPLR-FS-TARMA based modal parameter Monte Carlo estimates (30 realizations): (a) natural frequency estimates and (b) damping ratio estimates.

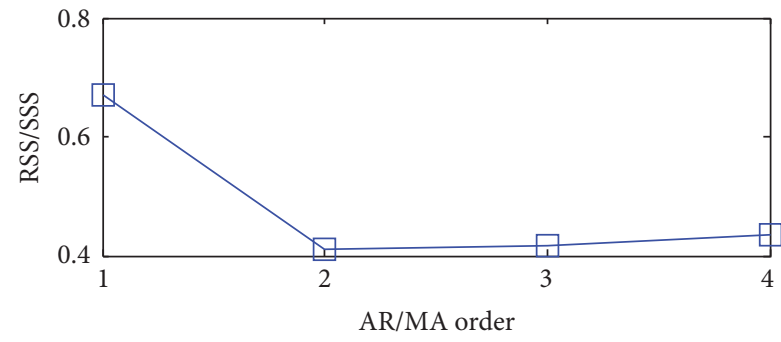

(a)

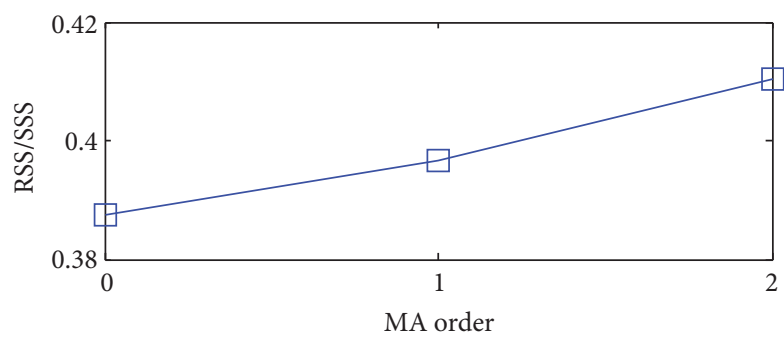

(b)

FIGURE 13: Exponentially weighted RPLR-TARMA model order selection: (a) AR order and (b) MA order.

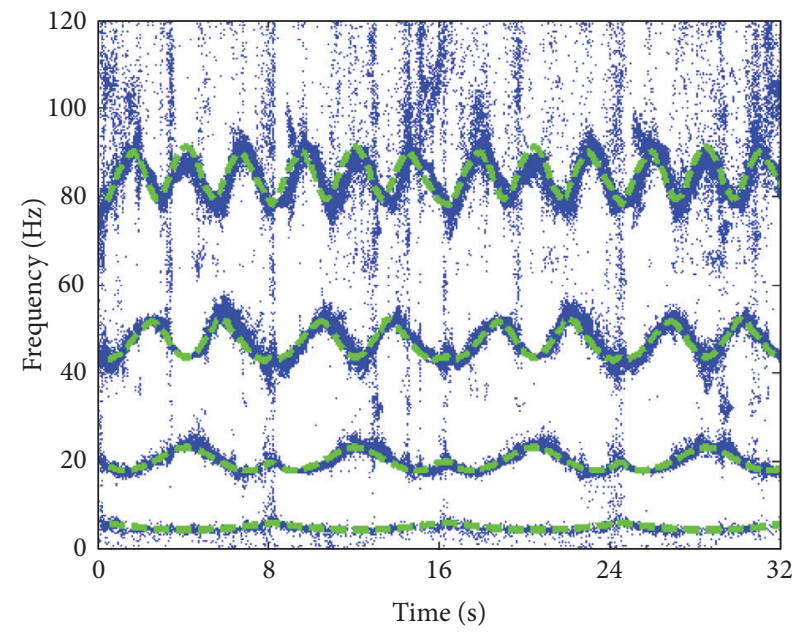

(a)
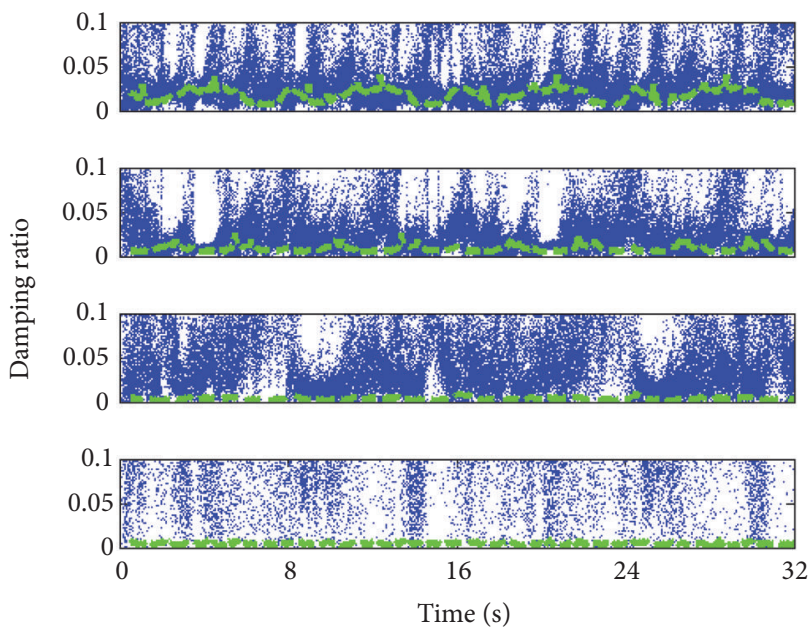

(b)

FIGURE 14: Exponentially weighted RPLR-TARMA based modal parameter Monte Carlo estimates (30 realizations): (a) natural frequency estimates and (b) damping ratio estimates. 


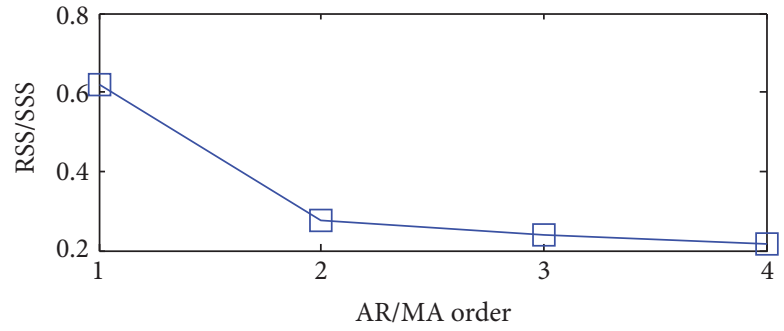

(a)

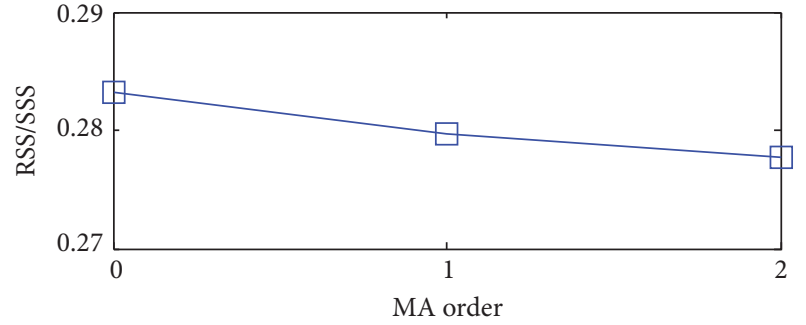

(b)

FIGURE 15: KRR-FS-TARMA model order selection: (a) AR order and (b) MA order.

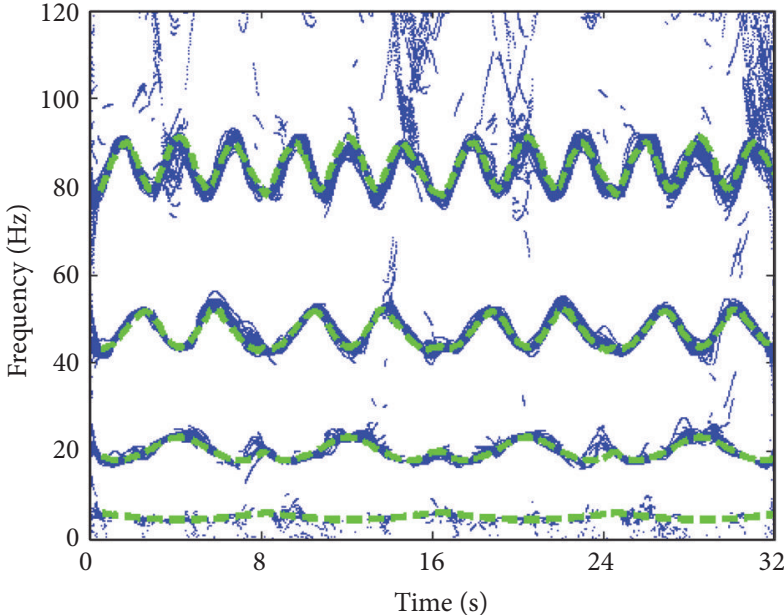

(a)
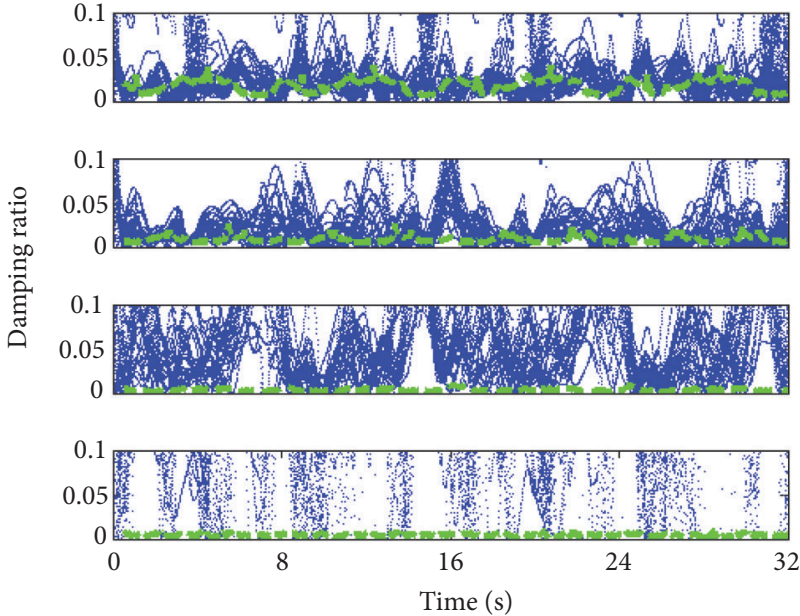

(b)

FIGURE 16: KRR-FS-TARMA based modal parameter Monte Carlo estimates (30 realizations): (a) natural frequency estimates and (b) damping ratio estimates.

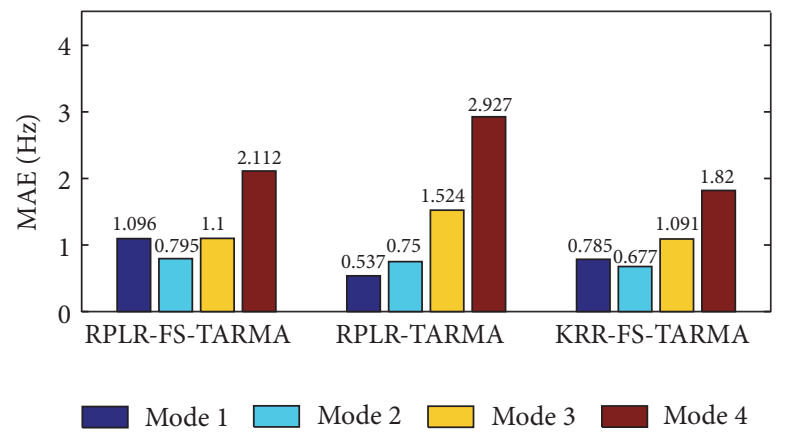

Figure 17: Monte Carlo comparison of different methods: MAE of natural frequency estimates.

The comparative assessments demonstrate the proposed KRR-FS-TARMA approach has superior achievable accuracy and enhanced tracking capability that are typical of the DPE methods but, at the same time, has improved flexibility of recursive estimation, which is typical of the UPE methods.

\section{Abbreviations}

AR: $\quad$ Autoregressive

MA: $\quad$ Moving average

TARMA: Time-dependent autoregressive moving average (model)
FS-TARMA: $\quad$ Functional series TARMA (model) RPLR-TARMA: Recursive pseudolinear regression TARMA (method)

RPLR-FS-TARMA: Recursive pseudolinear regression FS-TARMA (method)

KRR-FS-TARMA: Kernel ridge regression FS-TARMA (method)

UPE: $\quad$ Unstructured parameter evolution SPE: $\quad$ Stochastic parameter evolution DPE: Deterministic parameter evolution RSS: Residual sum of squares 
SSS: Series sum of squares

MAE: Mean absolute error.

\section{Competing Interests}

The authors declare that they have no competing interests.

\section{Acknowledgments}

The authors acknowledge the support from the China Scholarship Council and National Natural Science Foundation of China (Grant no. 11402022).

\section{References}

[1] S. W. Doebling, C. R. Farrar, and M. B. Prime, "A summary review of vibration-based damage identification methods," Shock and Vibration Digest, vol. 30, no. 2, pp. 91-105, 1998.

[2] E. P. Carden and P. Fanning, "Vibration based condition monitoring: a review," Structural Health Monitoring, vol. 3, no. 4, pp. 355-377, 2004.

[3] S. D. Fassois and J. S. Sakellariou, "Time-series methods for fault detection and identification in vibrating structures," Mathematic, Physical \& Engineering Sciences, vol. 365, pp. 411-448, 2007.

[4] W. Fan and P. Qiao, "Vibration-based damage identification methods: A Review And Comparative Study," Structural Health Monitoring, vol. 10, no. 1, pp. 83-111, 2011.

[5] M. D. Spiridonakos and S. D. Fassois, "An FS-TAR based method for vibration-response-based fault diagnosis in stochastic time-varying structures: experimental application to a pickand-place mechanism," Mechanical Systems and Signal Processing, vol. 38, no. 1, pp. 206-222, 2013.

[6] W. Heylen, S. Lammens, and P. Sas, Modal Analysis Theory and Testing, Katholieke Universiteit Leuven, Leuven, Belgium, 2007.

[7] H. D’Angelo, Linear Time-Varying Systems: Analysis and Synthesis, Allyn \& Bacon, Boston, Mass, USA, 1970.

[8] Y. S. Shmaliy, Continuous-Time Systems, Springer, Dordrecht, The Netherlands, 2007.

[9] L. Ljung, System Identification-Theory for the User, Prentice Hall, Upper Saddle River, NJ, USA, 2nd edition, 1999.

[10] M. Niedzwiecki, Identification of Time-Varying Processes, John Wiley \& Sons, New York, NY, USA, 2000.

[11] A. G. Poulimenos and S. D. Fassois, "Parametric time-domain methods for non-stationary random vibration modelling and analysis-a critical survey and comparison," Mechanical Systems and Signal Processing, vol. 20, no. 4, pp. 763-816, 2006.

[12] M. D. Spiridonakos and S. D. Fassois, "Non-stationary random vibration modelling and analysis via functional series timedependent ARMA (FS-TARMA) models-a critical survey," Mechanical Systems and Signal Processing, vol. 47, no. 1-2, pp. 175-224, 2014.

[13] M. D. Spiridonakos and S. D. Fassois, "Parametric identification of a time-varying structure based on vector vibration response measurements," Mechanical Systems and Signal Processing, vol. 23, no. 6, pp. 2029-2048, 2009.

[14] W. Yang, L. Liu, S.-D. Zhou, and Z.-S. Ma, "Moving Kriging shape function modeling of vector TARMA models for modal identification of linear time-varying structural systems," Journal of Sound and Vibration, vol. 354, pp. 254-277, 2015.
[15] X. Y. Xie and R. J. Evans, "Discrete time stochastic adaptive control for time-varying systems," IEEE Transactions on Automatic Control, vol. 29, no. 7, pp. 638-640, 1984.

[16] Z. Li, "Discrete-time adaptive control of deterministic fast timevarying systems," IEEE Transactions on Automatic Control, vol. 32, no. 5, pp. 444-447, 1987.

[17] M. Niedzwiecki, "Recursive functional series modeling estimators for identification of time-varying plants-more bad news than good?" IEEE Transactions on Automatic Control, vol. 35, no. 5, pp. 610-616, 1990.

[18] M. K. Tsatsanis and G. B. Giannakis, "Modelling and equalization of rapidly fading channels," International Journal of Adaptive Control and Signal Processing, vol. 10, no. 2-3, pp. 159-176, 1996.

[19] A. Joensen, H. Madsen, H. A. Nielsen, and T. S. Nielsen, “Tracking time-varying parameters with local regression," Automatica, vol. 36, no. 8, pp. 1199-1204, 2000.

[20] M. Niedzwiecki and T. Kłaput, "Fast recursive basis function estimators for identification of time-varying processes," IEEE Transactions on Signal Processing, vol. 50, no. 8, pp. 1925-1934, 2002.

[21] G. G. Walter, Wavelets and Other Orthogonal Systems with Applications, Taylor \& Francis, Boca Raton, Fla, USA, 1994.

[22] H. Lütkepohl, Handbook of Matrices, John Wiley \& Sons, New York, NY, USA, 1996.

[23] S. Yang, Y. Wu, and J. Xuan, Time Series Analysis in Engineering Application, Huazhong University of Science and Technology Press, Wuhan, China, 2nd edition, 2007.

[24] C. M. Bishop, Pattern Recognition and Machine Learning, Springer, New York, NY, USA, 2006.

[25] C. E. Rasmussen and C. K. Williams, Gaussian processes for machine learning, Adaptive Computation and Machine Learning, MIT Press, Cambridge, Massachusetts, USA, 2006.

[26] W. Liu, J. C. Príncipe, and S. Haykin, Kernel Adaptive Filtering: A Comprehensive Introduction, John Wiley \& Sons, New Jersey, NJ, USA, 2010.

[27] S. Van Vaerenbergh, J. Via, and I. Santamaría, "Nonlinear system identification using a new sliding-window Kernel RLS algorithm," Journal of Communications, vol. 2, no. 3, pp. 1-8, 2007.

[28] J. A. K. Suykens, M. Signoretto, and A. Argyriou, Regularization, Optimization, Kernels, and Support Vector Machines, CRC Press, Boca Raton, Fla, USA, 2014.

[29] N. Aronszajn, “Theory of reproducing kernels," Transactions of the American Mathematical Society, vol. 68, pp. 337-404, 1950.

[30] A. Poulimenos, M. Spiridonakos, and S. Fassois, "Parametric time-domain methods for non-stationary random vibration identification and analysis: an overview and comparison," in Proceedings of the International Conference on Noise and Vibration Engineering (ISMA '06), pp. 2885-2905, Leuven, Belgium, 2006.

[31] Z.-S. Ma, L. Liu, S.-D. Zhou, and W. Yang, "Modal parameter estimation of the coupled moving-mass and beam time-varying system," in Proceedings of the International Conference on Noise and Vibration Engineering (ISMA '14), pp. 587-596, Leuven, Belgium, 2014. 


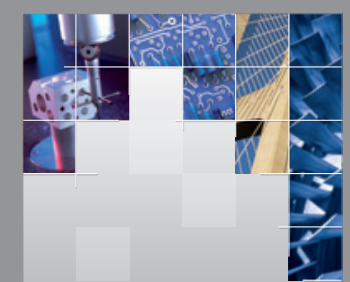

\section{Enfincering}
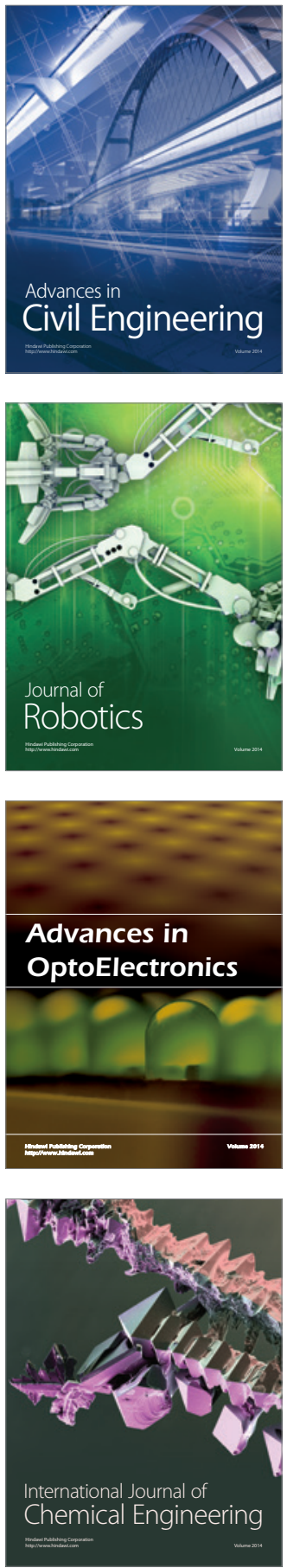

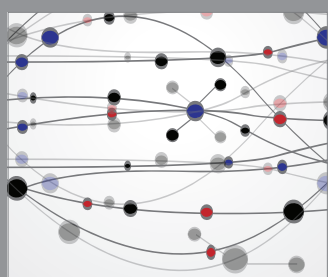

The Scientific World Journal

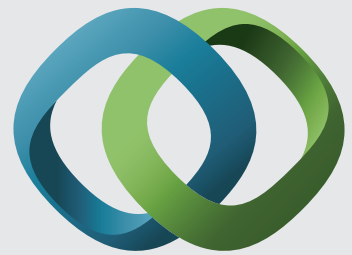

\section{Hindawi}

Submit your manuscripts at

https://www.hindawi.com
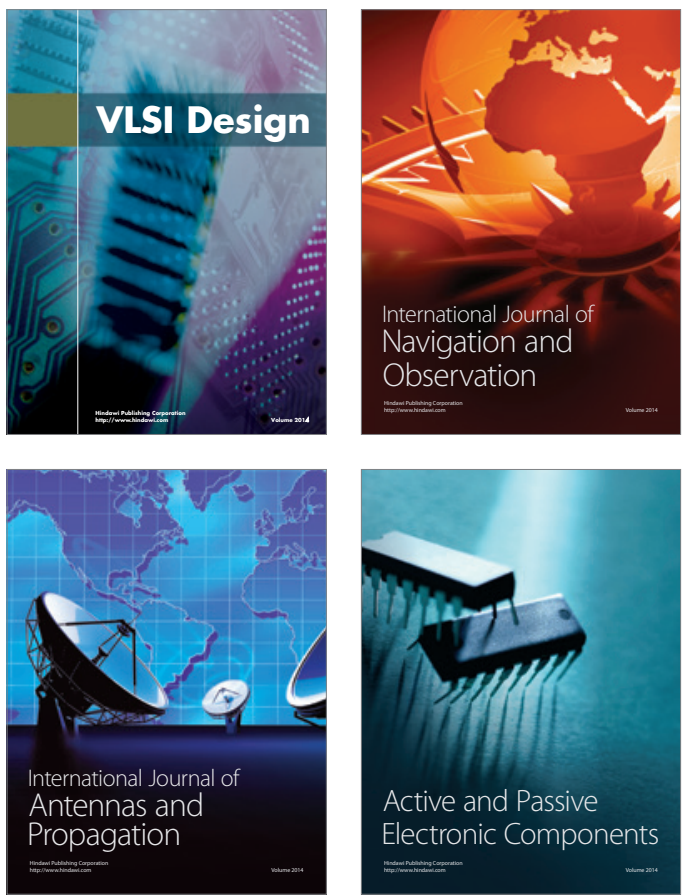
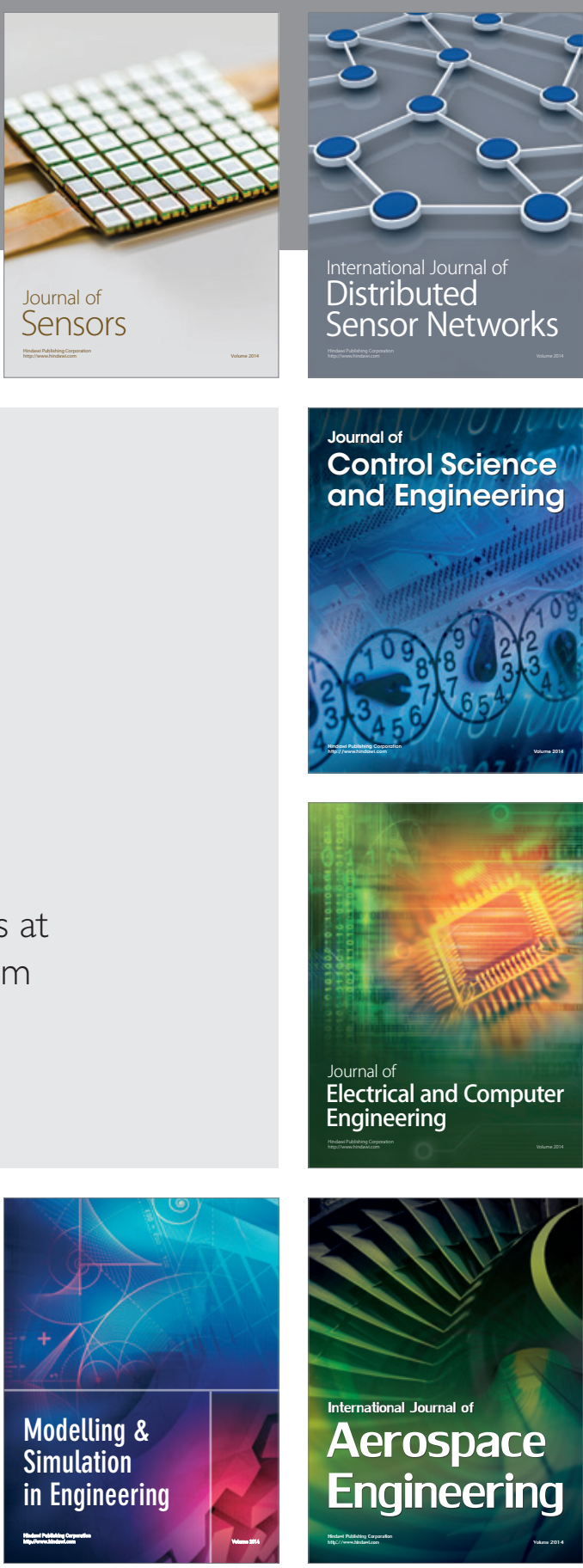

International Journal of

Distributed

Sensor Networks

$-$

Joumal of

Control Science

and Engineering
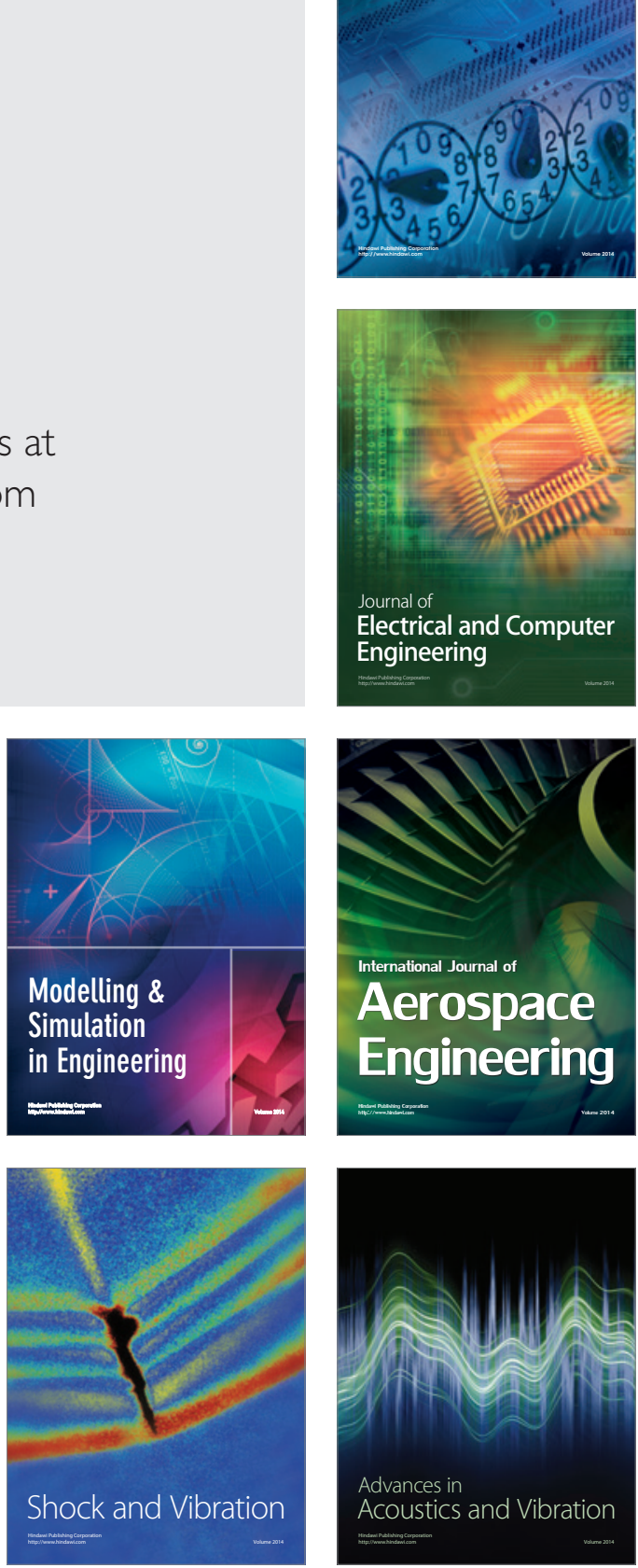